\title{
Oscillation Criteria for Delay and Advanced Differential Equations with Nonmonotone Arguments
}

\author{
George E. Chatzarakis ${ }^{1}$ and Tongxing $L i \mathbb{D}^{2,3}$ \\ ${ }^{1}$ Department of Electrical and Electronic Engineering Educators, School of Pedagogical and Technological Education (ASPETE), \\ N. Heraklio, 14121 Athens, Greece \\ ${ }^{2}$ School of Mathematical Sciences, Qufu Normal University, Qufu, Shandong 273165, China \\ ${ }^{3}$ School of Information Science and Engineering, Linyi University, Linyi, Shandong 276005, China
}

Correspondence should be addressed to Tongxing Li; litongx2007@163.com

Received 16 April 2017; Revised 27 June 2017; Accepted 6 March 2018; Published 18 April 2018

Academic Editor: Shanmugam Lakshmanan

Copyright (C) 2018 George E. Chatzarakis and Tongxing Li. This is an open access article distributed under the Creative Commons Attribution License, which permits unrestricted use, distribution, and reproduction in any medium, provided the original work is properly cited.

We study the oscillatory behavior of differential equations with nonmonotone deviating arguments and nonnegative coefficients. New oscillation criteria, involving limsup and liminf, are obtained based on an iterative method. Examples, numerically solved in MATLAB, are given to illustrate the applicability and strength of the obtained conditions over known ones.

\section{Introduction}

In mathematics, delay differential equations (DDEs) are that type of differential equations where the derivative of the unknown function, at a certain time, is given in terms of the values of the function, at previous times. DDEs are also referred in the literature as time-delay systems, systems with aftereffect or dead-time, hereditary systems, or equations with delay arguments.

Mathematical modelling involving DDEs is widely used for analysis and predictions in various areas of the life sciences, for example, population dynamics, epidemiology, immunology, physiology, neural networks. See, for example, [1-10] and the references cited therein. The time delays add to these models memory effects, taking into account the dependence of the model's present state on its past history [9]. The delay can be related to the duration of certain hidden processes, like the stages of the life cycle, the time between infection of a cell and the production of new viruses, the duration of the infectious period, the immune period, and so on.

In analogy, advanced differential equations (ADEs) are used in many applied problems where the evolution rate depends not only on the present, but also on the future.
While delays in DDEs represent the retrospective memory of the past, advances in ADEs represent the prospective memory of the future, accounting for the influence on the system of potential future actions, which are available, at the present time. For instance, population dynamics, economics problems, or mechanical control engineering are typical fields where such phenomena are thought to occur (see $[11,12]$ for details).

The earliest delay model in mathematical biology is Hutchinson's equation, in 1948 [6]. Hutchinson modified the classical logistic equation, with a delay term to incorporate hatching and maturation periods into the model and account for oscillations, in the population of Daphnia,

$$
y^{\prime}(t)=r y(t)\left(1-\frac{y(t-\tau)}{K}\right)
$$

where $y(t)$ denotes the size of the population, in the present time $t, y^{\prime}(t)$ describes the change of this size, at time $t, y(t-\tau)$ is the size, in some past time $t-\tau, \tau>0$ is the delay, representing the time for new eggs to hatch, and $r$ is the reproduction rate of the population, while $K$ is the carrying capacity, for the population. 
Many physiological processes, including the concentration of red blood cells, the concentration of $\mathrm{CO}_{2}$ in the blood, causing the observed periodic oscillations in the breathing frequency, and the production of new blood cells, in the bone marrow, exhibit oscillations and several DDE models have been proposed to model these processes.

Below, we present two applications indicating the relevance of the DDEs we study in this paper to real world problems. The two examples are taken from the areas of physiology and population dynamics.

Application 1 (blood cells production [9]). The production of red and white blood cells, in the bone marrow, is regulated by the level of oxygen, in the blood. A reduction in the number of cells in the blood, as a result of the loss of cells, causes the level of oxygen in the blood to decrease. When the level of oxygen in the blood decreases, a substance is released that in turn leads to the release of blood elements, from the bone marrow. Thus, the concentration $c(t)$ of cells in the blood stream, at any time $t$, changes according to the loss of cells and the release of new cells, from the bone marrow. But the bone marrow responds to a reduction in the number of blood cells and the decrease in the level of oxygen, with a delay that is in the order of 6 days. That means the release of new cells, into the blood stream, at time $t$, depends on the cell concentration, at an earlier time, namely, $t-\tau$, where $\tau$ is the delay with which the bone marrow responds to a reduced level of oxygen in the blood. The simplest model of the concentration of the cells in the blood stream can be described by the DDE

$$
c^{\prime}(t)=\lambda c(t-\tau)-\gamma c(t)
$$

where $\lambda$ represents the flux of cells into the blood stream, $\gamma$ is the death rate, and $\tau$ is the delay. All of them are positive constants. The solutions of the above equation exhibit similar oscillations to the actual oscillatory pattern observed in the concentration of cells in the blood stream.

Application 2. Imagine a biological population composed of adult and juvenile individuals. Let $N(t)$ denote the density of adults at time $t$. Assume that the length of the juvenile period is exactly $h$ units of time for each individual. Assume that adults produce offspring at a per capita rate $\alpha$ and that their probability per unit of time of dying is $\mu$. Assume that a newborn survives the juvenile period with probability $\rho$ and put $t=\alpha \rho$. Then the dynamics of $N$ can be described by the differential equation

$$
N^{\prime}(t)=-\mu N(t)+r N(t-h)
$$

which involves a nonlocal term, $r N(t-h)$ meaning that newborns become adults with some delay. So the time variation of the population density $N$ involves the current as well as the past values of $N$.

The use of DDEs, from the initial application, in population dynamics, has spread to every area of the life sciences: immunology, physiology, epidemiology, and cell growth. The original delay logistic equation has led to several new DDE forms, like Volterra's integrodifferential equations and neutral
DDEs [9], and several new models, from the delayed Hopfield model, in neural networks to the SIR model, in epidemiology [7]. More recently, the idea of state dependent delays has been introduced, involving "a delay that itself is governed by a differential equation that represents adaptation to the system's state" [9].

From the above review of DDEs, in the biological sciences, it is apparent that if DDEs are so extensively used in this area, this is because the dynamics of those equations, namely, the stability and oscillatory properties of the solutions of those equations, replicate the stability and oscillatory patterns, we actually observe in processes, in those areas. Thus, the study of the stability and oscillatory behavior of the solutions of DDEs has become the principal subject of the research on those equations. For more advanced treatises on oscillation theory, the reader is referred to [13-33].

In the paper, we consider a differential equation with delay argument of the form

$$
x^{\prime}(t)+p(t) x(\tau(t))=0, \quad t \geq t_{0}
$$

where $p$ is a function of nonnegative real numbers and $\tau$ is a function of positive real numbers such that

$$
\begin{aligned}
\tau(t) & <t, \quad t \geq t_{0}, \\
\lim _{t \rightarrow \infty} \tau(t) & =\infty
\end{aligned}
$$

By a solution of (E) we understand a continuously differentiable function defined on $\left[\tau\left(T_{0}\right), \infty\right)$ for some $T_{0} \geq t_{0}$ and such that (E) is satisfied for $t \geq T_{0}$. Such a solution is called oscillatory if it has arbitrarily large zeros, and otherwise it is called nonoscillatory. An equation is oscillatory if all its solutions oscillate.

A parallel problem to that of establishing oscillation criteria for the solutions of equation (E) is the one concerning the solutions of the advanced differential equation (ADE)

$$
x^{\prime}(t)-q(t) x(\sigma(t))=0, \quad t \geq t_{0}
$$

where $q$ is a function of nonnegative real numbers and $\sigma$ is a function of positive real numbers such that

$$
\sigma(t)>t, \quad t \geq t_{0}
$$

The objective of this paper is to consider the oscillatory dynamics of both delay and advanced differential equations, from the perspective of the qualitative analysis of those equations. In that framework, (i) we formulate new iterative oscillation conditions, for testing whether all solutions of a $\mathrm{DDE}$ of the form of (E) or an ADE of the form of $\left(\mathrm{E}^{\prime}\right)$ are oscillatory, (ii) we show that these tests significantly improve on all the previous, iterative, and noniterative oscillation criteria which, briefly, are reviewed in the Historical and Chronological Review, in Section 2, requiring fewer iterations to determine whether an equation of the considered form is oscillatory, and (iii) these criteria apply to a more general class of equations, having nonmonotone arguments $\tau(t)$ or $\sigma(t)$, in contrast to the large majority of the other studies where the criteria apply to equations with nondecreasing arguments. 
From this point onward, we will use the notation

$$
\begin{aligned}
& \alpha:=\liminf _{t \rightarrow \infty} \int_{\tau(t)}^{t} p(s) d s, \\
& \beta:=\liminf _{t \rightarrow \infty} \int_{t}^{\sigma(t)} q(s) d s, \\
& D(\omega):= \begin{cases}0, & \text { if } \omega>\frac{1}{e}, \\
\frac{1-\omega-\sqrt{1-2 \omega-\omega^{2}}}{2}, & \text { if } \omega \in\left[0, \frac{1}{e}\right],\end{cases} \\
& \mathrm{LD}:=\limsup _{t \rightarrow \infty}^{t} p(s) d s, \\
& \text { where } \tau(t) \text { is nondecreasing, } \\
& \text { LA }=\limsup _{t \rightarrow \infty} \int_{t}^{\sigma(t)} q(s) d s, \\
& \text { where } \sigma(t) \text { is nondecreasing. }
\end{aligned}
$$

\section{Historical and Chronological Review}

2.1. DDEs. The first systematic study for the oscillation of all solutions of equation (E) was made by Myškis in 1950 [31], when he proved that every solution of (E) oscillates, if

$$
\begin{array}{r}
\limsup _{t \rightarrow \infty}[t-\tau(t)]<\infty, \\
\liminf _{t \rightarrow \infty}[t-\tau(t)] \liminf _{t \rightarrow \infty} p(t)>\frac{1}{e} .
\end{array}
$$

In 1972, Ladas et al. [27] proved that if

$$
\mathrm{LD}>1
$$

then all solutions of (E) are oscillatory.

In 1982, Koplatadze and Chanturiya [24] improved (7) to

$$
\alpha>\frac{1}{e} .
$$

Regarding the constant $1 / e$ in (9), it should be remarked that if the inequality

$$
\int_{\tau(t)}^{t} p(s) d s \leq \frac{1}{e}
$$

holds eventually, then, according to [24], (E) has a nonoscillatory solution.

It is apparent that there is a gap between conditions (8) and (9), when

$$
\lim _{t \rightarrow \infty} \int_{\tau(t)}^{t} p(s) d s
$$

does not exist. How to fill this gap is an interesting problem which has been investigated by several authors. For example, in 2000, Jaroš and Stavroulakis [23] proved that if $\lambda_{0}$ is the smaller root of the equation $\lambda=e^{\alpha \lambda}$ and

$$
\mathrm{LD}>\frac{1+\ln \lambda_{0}}{\lambda_{0}}-D(\alpha),
$$

then all solutions of (E) oscillate.

Now we come to the general case where the argument $\tau(t)$ is nonmonotone. Set

$$
h(t):=\sup _{s \leq t} \tau(s), \quad t \geq t_{0} .
$$

Clearly, the function $h(t)$ is nondecreasing and $\tau(t) \leq h(t)<$ $t$, for all $t \geq t_{0}$.

In 1994, Koplatadze and Kvinikadze [25] proved that if

$$
\begin{aligned}
& \limsup _{t \rightarrow \infty} \int_{h(t)}^{t} p(s) \exp \left(\int_{h(s)}^{h(t)} p(u) \psi_{j}(u) d u\right) d s \\
& \quad>1-D(\alpha),
\end{aligned}
$$

where

$$
\begin{aligned}
& \psi_{1}(t)=0, \\
& \psi_{j}(t)=\exp \left(\int_{\tau(t)}^{t} p(u) \psi_{j-1}(u) d u\right), \quad j \geq 2,
\end{aligned}
$$

then all solutions of (E) oscillate.

In 2011, Braverman and Karpuz [14] proved that if

$$
\limsup _{t \rightarrow \infty} \int_{h(t)}^{t} p(s) \exp \left(\int_{\tau(s)}^{h(t)} p(u) d u\right) d s>1,
$$

then all solutions of (E) oscillate, while in 2014, Stavroulakis [32] improved (16) to

$$
\begin{aligned}
& \limsup _{t \rightarrow \infty} \int_{h(t)}^{t} p(s) \exp \left(\int_{\tau(s)}^{h(t)} p(u) d u\right) d s \\
& \quad>1-D(\alpha) .
\end{aligned}
$$

In 2016, El-Morshedy and Attia [30] proved that if

$$
\begin{aligned}
& \limsup _{t \rightarrow \infty}\left[\int_{g(t)}^{t} p_{n}(s) d s\right. \\
& \left.\quad+D(\alpha) \exp \left(\int_{g(t)}^{t} \sum_{j=0}^{n-1} p_{j}(s) d s\right)\right]>1,
\end{aligned}
$$

where $p_{0}(t)=p(t)$ and

$$
\begin{aligned}
& p_{n}(t) \\
& \quad=p_{n-1}(t) \int_{g(t)}^{t} p_{n-1}(s) \exp \left(\int_{g(s)}^{t} p_{n-1}(u) d u\right) d s,
\end{aligned}
$$


then all solutions of (E) are oscillatory. Here, $g(t)$ is a nondecreasing continuous function such that $\tau(t) \leq g(t) \leq t, t \geq$ $t_{1}$, for some $t_{1} \geq t_{0}$. Clearly, $g(t)$ is more general than $h(t)$ defined by (13). $\mathbb{N}$

Recently, Chatzarakis $[15,16]$ proved that if, for some $j \in$

$$
\limsup _{t \rightarrow \infty} \int_{h(t)}^{t} p(s) \exp \left(\int_{\tau(s)}^{h(t)} p_{j}(u) d u\right) d s>1
$$

or

$$
\begin{aligned}
& \underset{t \rightarrow \infty}{\limsup } \int_{h(t)}^{t} p(s) \exp \left(\int_{\tau(s)}^{h(t)} p_{j}(u) d u\right) d s \\
& \quad>1-D(\alpha)
\end{aligned}
$$

where

$$
\begin{aligned}
& p_{j}(t) \\
& \quad=p(t)\left[1+\int_{\tau(t)}^{t} p(s) \exp \left(\int_{\tau(s)}^{h(t)} p_{j-1}(u) d u\right) d s\right],
\end{aligned}
$$

with $p_{0}(t)=p(t)$, then all solutions of $(\mathrm{E})$ are oscillatory.

Lately, Chatzarakis [17] studied a more general form of (E); namely,

$$
x^{\prime}(t)+\sum_{i=1}^{m} p_{i}(t) x\left(\tau_{i}(t)\right)=0, \quad t \geq t_{0},
$$

and established sufficient oscillation conditions. Those conditions can lead to (20) and (21) when $m=1$.

\subsection{ADEs. By Theorem 2.4.3 [29], if}

$$
\mathrm{LA}>1 \text {, }
$$

then all solutions of $\left(\mathrm{E}^{\prime}\right)$ are oscillatory.

In 1984, Fukagai and Kusano [21] proved that if

$$
\beta>\frac{1}{e}
$$

then all solutions of $\left(\mathrm{E}^{\prime}\right)$ are oscillatory, while if

$$
\int_{t}^{\sigma(t)} q(s) d s \leq \frac{1}{e} \quad \text { for all sufficiently large } t,
$$

then $\left(E^{\prime}\right)$ has a nonoscillatory solution.

Assume that the argument $\sigma(t)$ is not necessarily monotone. Set

$$
\rho(t)=\inf _{s \geq t} \sigma(s), \quad t \geq t_{0}
$$

Clearly, the function $\rho(t)$ is nondecreasing and $\sigma(t) \geq \rho(t)>$ $t$, for all $t \geq t_{0}$.

In 2015, Chatzarakis and Öcalan [18] proved that if

$$
\limsup _{t \rightarrow \infty} \int_{t}^{\rho(t)} q(s) \exp \left(\int_{\rho(t)}^{\sigma(s)} q(u) d u\right) d s>1,
$$

or

$$
\liminf _{t \rightarrow \infty} \int_{t}^{\rho(t)} q(s) \exp \left(\int_{\rho(t)}^{\sigma(s)} q(u) d u\right) d s>\frac{1}{e},
$$

then all solutions of $\left(\mathrm{E}^{\prime}\right)$ are oscillatory. $\mathbb{N}$

Recently, Chatzarakis $[15,16]$ proved that if, for some $j \in$

$$
\limsup _{t \rightarrow \infty} \int_{t}^{\rho(t)} q(s) \exp \left(\int_{\rho(t)}^{\sigma(s)} q_{j}(u) d u\right) d s>1,
$$

or

$$
\begin{aligned}
& \limsup _{t \rightarrow \infty} \int_{t}^{\rho(t)} q(s) \exp \left(\int_{\rho(t)}^{\sigma(s)} q_{j}(u) d u\right) d s \\
& \quad>1-D(\beta),
\end{aligned}
$$

where

$$
\begin{array}{r}
q_{j}(t) \\
=q(t)\left[1+\int_{t}^{\sigma(t)} q(s) \exp \left(\int_{\rho(t)}^{\sigma(s)} q_{j-1}(u) d u\right) d s\right], \\
j \geq 1
\end{array}
$$

with $q_{0}(t)=q(t)$, then all solutions of $\left(\mathrm{E}^{\prime}\right)$ oscillate.

Lately, Chatzarakis [17] studied a more general form of $\left(\mathrm{E}^{\prime}\right)$, namely,

$$
x^{\prime}(t)-\sum_{i=1}^{m} q_{i}(t) x\left(\sigma_{i}(t)\right)=0, \quad t \geq t_{0},
$$

and established sufficient oscillation conditions. Those conditions can lead to (30) and (31) when $m=1$.

\section{Main Results}

3.1. DDEs. In our main results, we state theorems, establishing new sufficient oscillation conditions. For the proofs of those theorems, we use the following lemmas.

Lemma 3 (see [19, Lemma 2.1.1]). Assume that $h(t)$ is defined by (13). Then

$$
\alpha:=\liminf _{t \rightarrow \infty} \int_{\tau(t)}^{t} p(s) d s=\liminf _{t \rightarrow \infty} \int_{h(t)}^{t} p(s) d s .
$$

Lemma 4 (see [19, Lemma 2.1.3]). Assume that $h(t)$ is defined by (13), $\alpha \in(0,1 / e]$, and $x(t)$ is an eventually positive solution of $(E)$. Then

$$
\liminf _{t \rightarrow \infty} \frac{x(t)}{x(h(t))} \geq D(\alpha)
$$

Lemma 5 (see [26]). Assume that $h(t)$ is defined by (13), $\alpha \in$ $(0,1 / e]$, and $x(t)$ is an eventually positive solution of $(E)$. Then

$$
\liminf _{t \rightarrow \infty} \frac{x(h(t))}{x(t)} \geq \lambda_{0}
$$

where $\lambda_{0}$ is the smaller root of the equation $\lambda=e^{\alpha \lambda}$. 
Theorem 6. Let $h(t)$ be defined by (13) and for some $j \in \mathbb{N}$

$$
\begin{aligned}
& \underset{t \rightarrow \infty}{\limsup } \int_{h(t)}^{t} p(s) \\
& \quad \cdot \exp \left(\int_{\tau(s)}^{h(t)} p(u) \exp \left(\int_{\tau(u)}^{u} P_{j}(\xi) d \xi\right) d u\right) d s \\
& \quad>1
\end{aligned}
$$

where

$$
\begin{aligned}
& P_{j}(t)=p(t)\left[1+\int_{\tau(t)}^{t} p(s)\right. \\
& \left.\quad \cdot \exp \left(\int_{\tau(s)}^{t} p(u) \exp \left(\int_{\tau(u)}^{u} P_{j-1}(\xi) d \xi\right) d u\right) d s\right]
\end{aligned}
$$

with $P_{0}(t)=\lambda_{0} p(t)$, and let $\lambda_{0}$ be the smaller root of the equation $\lambda=e^{\alpha \lambda}$. Then all solutions of $(E)$ oscillate.

Proof. Assume, for the sake of contradiction, that there exists a nonoscillatory solution $x(t)$ of $(\mathrm{E})$. Since $-x(t)$ is also a solution of $(E)$, we can confine our discussion only to the case where the solution $x(t)$ is eventually positive. Then there exists a real number $t_{1}>t_{0}$ such that $x(t), x(\tau(t))>0$ for all $t \geq t_{1}$. Thus, from (E) we have

$$
x^{\prime}(t)=-p(t) x(\tau(t)) \leq 0 \quad \forall t \geq t_{1},
$$

which means that $x(t)$ is an eventually nonincreasing function of positive numbers. Taking into account the fact that $\tau(t) \leq h(t),(\mathrm{E})$ implies that

$$
x^{\prime}(t)+p(t) x(h(t)) \leq 0, \quad t \geq t_{1} .
$$

Observe that (36) implies that, for each $\epsilon>0$, there exists a real number $t_{\epsilon}$ such that

$$
\frac{x(h(t))}{x(t)}>\lambda_{0}-\epsilon \quad \forall t \geq t_{\epsilon} \geq t_{1} .
$$

Combining inequalities (40) and (41), we obtain

$$
x^{\prime}(t)+p(t)\left(\lambda_{0}-\epsilon\right) x(t) \leq 0, \quad t \geq t_{\epsilon},
$$

or

$$
x^{\prime}(t)+P_{0}(t, \epsilon) x(t) \leq 0, \quad t \geq t_{\epsilon},
$$

where

$$
P_{0}(t, \epsilon)=p(t)\left(\lambda_{0}-\epsilon\right)
$$

Applying the Grönwall inequality in (43), we conclude that

$$
x(s) \geq x(t) \exp \left(\int_{s}^{t} P_{0}(\xi, \epsilon) d \xi\right), \quad t \geq s \geq t_{\epsilon} .
$$

Now we divide (E) by $x(t)>0$ and integrate on $[s, t]$, so

$$
-\int_{s}^{t} \frac{x^{\prime}(u)}{x(u)} d u=\int_{s}^{t} p(u) \frac{x(\tau(u))}{x(u)} d u,
$$

or

$$
\ln \frac{x(s)}{x(t)}=\int_{s}^{t} p(u) \frac{x(\tau(u))}{x(u)} d u, \quad t \geq s \geq t_{\epsilon} .
$$

Since $\tau(u)<u$, equality (47) gives

$$
\begin{aligned}
\ln \frac{x(s)}{x(t)} & =\int_{s}^{t} p(u) \frac{x(\tau(u))}{x(u)} d u \\
& \geq \int_{s}^{t} p(u) \frac{x(u)}{x(u)} \exp \left(\int_{\tau(u)}^{u} P_{0}(\xi, \epsilon) d \xi\right) d u \\
& =\int_{s}^{t} p(u) \exp \left(\int_{\tau(u)}^{u} P_{0}(\xi, \epsilon) d \xi\right) d u,
\end{aligned}
$$

or

$$
x(s)
$$

Substituting $\tau(s)$ for $s$ in (49), we get

$$
\begin{aligned}
& x(\tau(s)) \\
& \geq x(t) \exp \left(\int_{\tau(s)}^{t} p(u) \exp \left(\int_{\tau(u)}^{u} P_{0}(\xi, \epsilon) d \xi\right) d u\right) .
\end{aligned}
$$

Integrating (E) from $\tau(t)$ to $t$, we have

$$
x(t)-x(\tau(t))+\int_{\tau(t)}^{t} p(s) x(\tau(s)) d s=0 .
$$

Combining (50) and (51), we obtain

$$
\begin{aligned}
& x(t)-x(\tau(t))+x(t) \int_{\tau(t)}^{t} p(s) \\
& \quad \cdot \exp \left(\int_{\tau(s)}^{t} p(u) \exp \left(\int_{\tau(u)}^{u} P_{0}(\xi, \epsilon) d \xi\right) d u\right) d s \\
& \quad \leq 0 .
\end{aligned}
$$

Multiplying inequality (52) by $p(t)$, we find

$$
\begin{aligned}
& p(t) x(t)-p(t) x(\tau(t))+p(t) x(t) \int_{\tau(t)}^{t} p(s) \\
& \quad \cdot \exp \left(\int_{\tau(s)}^{t} p(u) \exp \left(\int_{\tau(u)}^{u} P_{0}(\xi, \epsilon) d \xi\right) d u\right) d s \\
& \quad \leq 0
\end{aligned}
$$

which, in view of $(\mathrm{E})$, becomes

$$
\begin{aligned}
& x^{\prime}(t)+p(t) x(t)+p(t) x(t) \int_{\tau(t)}^{t} p(s) \\
& \cdot \exp \left(\int_{\tau(s)}^{t} p(u) \exp \left(\int_{\tau(u)}^{u} P_{0}(\xi, \epsilon) d \xi\right) d u\right) d s
\end{aligned}
$$


Hence, for sufficiently large $t$,

$$
\begin{aligned}
& x^{\prime}(t)+p(t)\left[1+\int_{\tau(t)}^{t} p(s)\right. \\
& \left.\cdot \exp \left(\int_{\tau(s)}^{t} p(u) \exp \left(\int_{\tau(u)}^{u} P_{0}(\xi, \epsilon) d \xi\right) d u\right) d s\right] \\
& \cdot x(t) \leq 0
\end{aligned}
$$

or

$$
x^{\prime}(t)+P_{1}(t, \epsilon) x(t) \leq 0,
$$

where

$$
\begin{aligned}
& P_{1}(t, \epsilon)=p(t)\left[1+\int_{\tau(t)}^{t} p(s)\right. \\
& \left.\quad \cdot \exp \left(\int_{\tau(s)}^{t} p(u) \exp \left(\int_{\tau(u)}^{u} P_{0}(\xi, \epsilon) d \xi\right) d u\right) d s\right] .
\end{aligned}
$$

Clearly (56) resembles (43), if we replace $P_{0}$ by $P_{1}$. Thus, integrating (56) on $[s, t]$ yields

$$
x(s) \geq x(t) \exp \left(\int_{s}^{t} P_{1}(\xi, \epsilon) d \xi\right) .
$$

Repeating steps (45) through (50), we can see that $x$ satisfies the inequality

$$
\begin{aligned}
& x(\tau(s)) \\
& \quad \geq x(t) \exp \left(\int_{\tau(s)}^{t} p(u) \exp \left(\int_{\tau(u)}^{u} P_{1}(\xi, \epsilon) d \xi\right) d u\right) .
\end{aligned}
$$

Combining now (51) and (59), we obtain

$$
\begin{aligned}
& x(t)-x(\tau(t))+x(t) \int_{\tau(t)}^{t} p(s) \\
& \quad \cdot \exp \left(\int_{\tau(s)}^{t} p(u) \exp \left(\int_{\tau(u)}^{u} P_{1}(\xi, \epsilon) d \xi\right) d u\right) d s \\
& \quad \leq 0 .
\end{aligned}
$$

Multiplying inequality (60) by $p(t)$, as before, we find

$$
\begin{aligned}
& x^{\prime}(t)+p(t)\left[1+\int_{\tau(t)}^{t} p(s)\right. \\
& \left.\quad \cdot \exp \left(\int_{\tau(s)}^{t} p(u) \exp \left(\int_{\tau(u)}^{u} P_{1}(\xi, \epsilon) d \xi\right) d u\right) d s\right] \\
& \cdot x(t) \leq 0 .
\end{aligned}
$$

Therefore, for sufficiently large $t$, we have

$$
x^{\prime}(t)+P_{2}(t, \epsilon) x(t) \leq 0
$$

where

$$
\begin{aligned}
& P_{2}(t, \epsilon)=p(t)\left[1+\int_{\tau(t)}^{t} p(s)\right. \\
& \left.\quad \cdot \exp \left(\int_{\tau(s)}^{t} p(u) \exp \left(\int_{\tau(u)}^{u} P_{1}(\xi, \epsilon) d \xi\right) d u\right) d s\right]
\end{aligned}
$$

It becomes apparent, now, that, by repeating the above steps, we can build inequalities on $x^{\prime}(t)$ with progressively higher indices $P_{j}(t, \epsilon), j \in \mathbb{N}$. In general, for sufficiently large $t$, the positive solution $x(t)$ satisfies the inequality

$$
x^{\prime}(t)+P_{j}(t, \epsilon) x(t) \leq 0, \quad j \in \mathbb{N},
$$

where

$$
\begin{aligned}
& P_{j}(t, \epsilon)=p(t)\left[1+\int_{\tau(t)}^{t} p(s)\right. \\
& \left.\quad \cdot \exp \left(\int_{\tau(s)}^{t} p(u) \exp \left(\int_{\tau(u)}^{u} P_{j-1}(\xi, \epsilon) d \xi\right) d u\right) d s\right] .
\end{aligned}
$$

Proceeding to final step, we recall that $h(t)$, defined by (13), is a nondecreasing function. Since $\tau(s) \leq h(s) \leq h(t)$, we have

$$
\begin{aligned}
& x(\tau(s)) \geq x(h(t)) \\
& \cdot \exp \left(\int_{\tau(s)}^{h(t)} p(u) \exp \left(\int_{\tau(u)}^{u} P_{j}(\xi, \epsilon) d \xi\right) d u\right) .
\end{aligned}
$$

Hence

$$
\begin{aligned}
& x(t)-x(h(t))+x(h(t)) \int_{h(t)}^{t} p(s) \\
& \quad \cdot \exp \left(\int_{\tau(s)}^{h(t)} p(u) \exp \left(\int_{\tau(u)}^{u} P_{j}(\xi, \epsilon) d \xi\right) d u\right) d s \\
& \quad \leq 0,
\end{aligned}
$$

or

$$
\begin{aligned}
& x(h(t)) {\left[\int_{h(t)}^{t} p(s)\right.} \\
& \cdot \exp \left(\int_{\tau(s)}^{h(t)} p(u) \exp \left(\int_{\tau(u)}^{u} P_{j}(\xi, \epsilon) d \xi\right) d u\right) d s \\
&-1]<0 .
\end{aligned}
$$

Thus

$$
\begin{aligned}
& \int_{h(t)}^{t} p(s) \\
& \quad \cdot \exp \left(\int_{\tau(s)}^{h(t)} p(u) \exp \left(\int_{\tau(u)}^{u} P_{j}(\xi, \epsilon) d \xi\right) d u\right) d s \\
& \quad-1<0 .
\end{aligned}
$$

Taking the limit as $t \rightarrow \infty$, we have

$$
\begin{aligned}
& \limsup _{t \rightarrow \infty} \int_{h(t)}^{t} p(s) \\
& \quad \cdot \exp \left(\int_{\tau(s)}^{h(t)} p(u) \exp \left(\int_{\tau(u)}^{u} P_{j}(\xi, \epsilon) d \xi\right) d u\right) d s
\end{aligned}
$$


Since $\epsilon$ may be taken arbitrarily small, this inequality contradicts (37).

This completes the proof of the theorem.

Theorem 7. Let $h(t)$ be defined by (13) and $\alpha \in(0,1 / e]$. If for some $j \in \mathbb{N}$

$$
\begin{aligned}
& \limsup _{t \rightarrow \infty} \int_{h(t)}^{t} p(s) \\
& \quad \cdot \exp \left(\int_{\tau(s)}^{h(t)} p(u) \exp \left(\int_{\tau(u)}^{u} P_{j}(\xi) d \xi\right) d u\right) d s \\
& \quad>1-D(\alpha)
\end{aligned}
$$

where $P_{j}$ is defined by (38), then all solutions of $(E)$ oscillate.

Proof. Assume $x$ is an eventually positive solution of (E). Clearly, (67) is satisfied for sufficiently large $t$. Thus,

$$
\begin{aligned}
& \int_{h(t)}^{t} p(s) \\
& \quad \cdot \exp \left(\int_{\tau(s)}^{h(t)} p(u) \exp \left(\int_{\tau(u)}^{u} P_{j}(\xi, \epsilon) d \xi\right) d u\right) d s \\
& \quad \leq 1-\frac{x(t)}{x(h(t))},
\end{aligned}
$$

which implies that

$$
\begin{aligned}
& \limsup _{t \rightarrow \infty} \int_{h(t)}^{t} p(s) \\
& \quad \cdot \exp \left(\int_{\tau(s)}^{h(t)} p(u) \exp \left(\int_{\tau(u)}^{u} P_{j}(\xi, \epsilon) d \xi\right) d u\right) d s \\
& \quad \leq 1-\liminf _{t \rightarrow \infty} \frac{x(t)}{x(h(t))} .
\end{aligned}
$$

Using Lemmas 3 and 4, it is evident that inequality (35) is satisfied. Thus, (73) leads to

$$
\begin{aligned}
& \limsup _{t \rightarrow \infty} \int_{h(t)}^{t} p(s) \\
& \quad \cdot \exp \left(\int_{\tau(s)}^{h(t)} p(u) \exp \left(\int_{\tau(u)}^{u} P_{j}(\xi, \epsilon) d \xi\right) d u\right) d s \\
& \quad \leq 1-D(\alpha) .
\end{aligned}
$$

Since $\epsilon$ may be taken arbitrarily small, this inequality contradicts (71).

This completes the proof of the theorem.
Theorem 8. Let $h(t)$ be defined by (13) and $\alpha \in(0,1 / e]$. If for some $j \in \mathbb{N}$

$$
\begin{aligned}
& \limsup _{t \rightarrow \infty} \int_{h(t)}^{t} p(s) \\
& \quad \cdot \exp \left(\int_{\tau(s)}^{t} p(u) \exp \left(\int_{\tau(u)}^{u} P_{j}(\xi) d \xi\right) d u\right) d s \\
& >\frac{1}{D(\alpha)}
\end{aligned}
$$

where $P_{j}$ is defined by (38), then all solutions of $(E)$ oscillate.

Proof. Assume $x$ is an eventually positive solution of (E). Then, as in the proof of Theorem 6 , for sufficiently large $t$, we conclude that

$$
\begin{aligned}
& x(\tau(s)) \\
& \geq x(t) \exp \left(\int_{\tau(s)}^{t} p(u) \exp \left(\int_{\tau(u)}^{u} P_{j}(\xi, \epsilon) d \xi\right) d u\right) .
\end{aligned}
$$

Integrating (E) from $h(t)$ to $t$ and using (76), we obtain

$$
\begin{aligned}
& x(t)-x(h(t))+\int_{h(t)}^{t} p(s) x(t) \\
& \quad \cdot \exp \left(\int_{\tau(s)}^{t} p(u) \exp \left(\int_{\tau(u)}^{u} P_{j}(\xi, \epsilon) d \xi\right) d u\right) d s \\
& \quad \leq 0,
\end{aligned}
$$

or

$$
\begin{aligned}
& -x(h(t))+\int_{h(t)}^{t} p(s) x(t) \\
& \quad \cdot \exp \left(\int_{\tau(s)}^{t} p(u) \exp \left(\int_{\tau(u)}^{u} P_{j}(\xi, \epsilon) d \xi\right) d u\right) d s \\
& \quad<0 .
\end{aligned}
$$

Hence

$$
\begin{aligned}
& x(h(t)) {\left[\frac{x(t)}{x(h(t))} \int_{h(t)}^{t} p(s)\right.} \\
& \cdot \exp \left(\int_{\tau(s)}^{t} p(u) \exp \left(\int_{\tau(u)}^{u} P_{j}(\xi, \epsilon) d \xi\right) d u\right) d s \\
&-1]<0,
\end{aligned}
$$

which yields, for all sufficiently large $t$,

$$
\begin{aligned}
& \int_{h(t)}^{t} p(s) \\
& \quad \cdot \exp \left(\int_{\tau(s)}^{t} p(u) \exp \left(\int_{\tau(u)}^{u} P_{j}(\xi, \epsilon) d \xi\right) d u\right) d s \\
& \quad<\frac{x(h(t))}{x(t)}
\end{aligned}
$$


and consequently

$$
\begin{aligned}
& \limsup _{t \rightarrow \infty} \int_{h(t)}^{t} p(s) \\
& \quad \cdot \exp \left(\int_{\tau(s)}^{t} p(u) \exp \left(\int_{\tau(u)}^{u} P_{j}(\xi, \epsilon) d \xi\right) d u\right) d s \\
& \quad \leq \limsup _{t \rightarrow \infty} \frac{x(h(t))}{x(t)} .
\end{aligned}
$$

Taking into account the fact that (35) is satisfied, inequality (81) leads to

$$
\begin{aligned}
& \limsup _{t \rightarrow \infty} \int_{h(t)}^{t} p(s) \\
& \quad \cdot \exp \left(\int_{\tau(s)}^{t} p(u) \exp \left(\int_{\tau(u)}^{u} P_{j}(\xi, \epsilon) d \xi\right) d u\right) d s \\
& \quad \leq \frac{1}{D(\alpha)},
\end{aligned}
$$

which contradicts (75), when $\epsilon \rightarrow 0$.

This completes the proof of the theorem.

Theorem 9. Let $h(t)$ be defined by (13) and $\alpha \in(0,1 / e]$. If for some $j \in \mathbb{N}$

$$
\begin{aligned}
& \limsup _{t \rightarrow \infty} \int_{h(t)}^{t} p(s) \\
& \cdot \exp \left(\int_{\tau(s)}^{h(s)} p(u) \exp \left(\int_{\tau(u)}^{u} P_{j}(\xi) d \xi\right) d u\right) d s \\
& \quad>\frac{1+\ln \lambda_{0}}{\lambda_{0}}-D(\alpha)
\end{aligned}
$$

where $P_{j}$ is defined by (38) and $\lambda_{0}$ is the smaller root of the equation $\lambda=e^{\alpha \lambda}$, then all solutions of $(E)$ oscillate.

Proof. Let $x$ be an eventually positive solution of (E). As in the proof of Theorem 8, we can show that (76) holds; namely,

$$
\begin{aligned}
& x(\tau(s)) \\
& \quad \geq x(t) \exp \left(\int_{\tau(s)}^{t} p(u) \exp \left(\int_{\tau(u)}^{u} P_{j}(\xi, \epsilon) d \xi\right) d u\right) .
\end{aligned}
$$

Since $\tau(s) \leq h(s)$, inequality (84) gives

$$
\begin{aligned}
x(\tau(s)) & \geq x(h(s)) \\
\cdot \exp & \left(\int_{\tau(s)}^{h(s)} p(u) \exp \left(\int_{\tau(u)}^{u} P_{j}(\xi, \epsilon) d \xi\right) d u\right) .
\end{aligned}
$$

By Lemma 5, for each $\epsilon>0$, there exists a real number $t_{\epsilon}$ such that

$$
\frac{x(h(t))}{x(t)}>\lambda_{0}-\epsilon \quad \forall t \geq t_{\epsilon} \geq t_{1} .
$$

Note that, by the nondecreasing nature of the function $x(h(t)) / x(s)$ in $s$, it holds

$$
\begin{aligned}
1=\frac{x(h(t))}{x(h(t))} \leq \frac{x(h(t))}{x(s)} \leq \frac{x(h(t))}{x(t)} & \\
& t_{\epsilon} \leq h(t) \leq s \leq t .
\end{aligned}
$$

In particular, for $\epsilon \in\left(0, \lambda_{0}-1\right)$, by continuity, we conclude that there exists a real number $t^{*} \in(h(t), t]$ satisfying

$$
1<\lambda_{0}-\epsilon=\frac{x(h(t))}{x\left(t^{*}\right)} .
$$

Integrating (E) from $t^{*}$ to $t$ and using (85), we obtain

$$
\begin{aligned}
& x(t)-x\left(t^{*}\right)+x(h(t)) \int_{t^{*}}^{t} p(s) \\
& \quad \cdot \exp \left(\int_{\tau(s)}^{h(s)} p(u) \exp \left(\int_{\tau(u)}^{u} P_{j}(\xi, \epsilon) d \xi\right) d u\right) d s
\end{aligned}
$$$$
\leq 0
$$

or

$$
\begin{aligned}
& \int_{t^{*}}^{t} p(s) \exp \left(\int_{\tau(s)}^{h(s)} p(u)\right. \\
& \left.\cdot \exp \left(\int_{\tau(u)}^{u} P_{j}(\xi, \epsilon) d \xi\right) d u\right) d s \\
& \quad \leq \frac{x\left(t^{*}\right)}{x(h(t))}-\frac{x(t)}{x(h(t))}
\end{aligned}
$$

Using (88) and Lemma 4, we deduce that, for the $\epsilon$ considered, there exists a real number $t_{\epsilon}^{\prime} \geq t_{\epsilon}$ such that

$$
\begin{aligned}
& \int_{t^{*}}^{t} p(s) \\
& \quad \cdot \exp \left(\int_{\tau(s)}^{h(s)} p(u) \exp \left(\int_{\tau(u)}^{u} P_{j}(\xi, \epsilon) d \xi\right) d u\right) d s \\
& \quad<\frac{1}{\lambda_{0}-\epsilon}-D(\alpha)+\epsilon
\end{aligned}
$$

for $t \geq t_{\epsilon}^{\prime}$.

Dividing (E) by $x(t)$, integrating from $h(t)$ to $t^{*}$, and using (85), we deduce that

$$
\begin{aligned}
& \int_{h(t)}^{t^{*}} p(s) \\
& \quad \cdot \frac{x(h(s))}{x(s)} \exp \left(\int_{\tau(s)}^{h(s)} p(u) \exp \left(\int_{\tau(u)}^{u} P_{j}(\xi, \epsilon) d \xi\right) d u\right) d s \\
& \quad \leq-\int_{h(t)}^{t^{*}} \frac{x^{\prime}(s)}{x(s)} d s .
\end{aligned}
$$


Clearly, by means of (36), $x(h(s)) / x(s)>\lambda_{0}-\epsilon$, for $s \geq h(t) \geq$ $t_{\epsilon}^{\prime}$. Hence, for all sufficiently large $t$, we conclude that

$$
\begin{aligned}
& \left(\lambda_{0}-\epsilon\right) \int_{h(t)}^{t^{*}} p(s) \\
& \cdot \exp \left(\int_{\tau(s)}^{h(s)} p(u) \exp \left(\int_{\tau(u)}^{u} P_{j}(\xi, \epsilon) d \xi\right) d u\right) d s \\
& <-\int_{h(t)}^{t^{*}} \frac{x^{\prime}(s)}{x(s)} d s
\end{aligned}
$$

or

$$
\begin{aligned}
& \int_{h(t)}^{t^{*}} p(s) \\
& \cdot \exp \left(\int_{\tau(s)}^{h(s)} p(u) \exp \left(\int_{\tau(u)}^{u} P_{j}(\xi, \epsilon) d \xi\right) d u\right) d s \\
& \quad<-\frac{1}{\lambda_{0}-\epsilon} \int_{h(t)}^{t^{*}} \frac{x^{\prime}(s)}{x(s)} d s=\frac{1}{\lambda_{0}-\epsilon} \ln \frac{x(h(t))}{x\left(t^{*}\right)} \\
& \quad=\frac{\ln \left(\lambda_{0}-\epsilon\right)}{\lambda_{0}-\epsilon}
\end{aligned}
$$

that is,

$$
\begin{aligned}
& \int_{h(t)}^{t^{*}} p(s) \\
& \quad \cdot \exp \left(\int_{\tau(s)}^{h(s)} p(u) \exp \left(\int_{\tau(u)}^{u} P_{j}(\xi, \epsilon) d \xi\right) d u\right) d s \\
& \quad<\frac{\ln \left(\lambda_{0}-\epsilon\right)}{\lambda_{0}-\epsilon} .
\end{aligned}
$$

$$
\ln \left(\frac{x(h(t))}{x(t)}\right)=\int_{h(t)}^{t} p(s) \frac{x(\tau(s))}{x(s)} d s \quad \forall t \geq t_{2} \geq t_{1},
$$

from which, in view of $\tau(s) \leq h(s)$ and (85), we get

$$
\begin{aligned}
& \ln \left(\frac{x(h(t))}{x(t)}\right) \geq \int_{h(t)}^{t} p(s) \\
& \quad \frac{x(h(s))}{x(s)} \exp \left(\int_{\tau(s)}^{h(s)} p(u) \exp \left(\int_{\tau(u)}^{u} P_{j}(\xi, \epsilon) d \xi\right) d u\right) d s .
\end{aligned}
$$

Since $x$ is nonincreasing and $h(s)<s$, inequality (99) becomes

$$
\begin{aligned}
& \ln \left(\frac{x(h(t))}{x(t)}\right) \geq \int_{h(t)}^{t} p(s) \\
& \quad \cdot \exp \left(\int_{\tau(s)}^{h(s)} p(u) \exp \left(\int_{\tau(u)}^{u} P_{j}(\xi, \epsilon) d \xi\right) d u\right) d s .
\end{aligned}
$$

From (97), it is clear that there exists a constant $c>0$ such that

$$
\begin{aligned}
& \int_{h(t)}^{t} p(s) \\
& \quad \cdot \exp \left(\int_{\tau(s)}^{h(s)} p(u) \exp \left(\int_{\tau(u)}^{u} P_{j}(\xi) d \xi\right) d u\right) d s \\
& \quad \geq c>\frac{1}{e} .
\end{aligned}
$$

Choose $c^{\prime}$ such that $c>c^{\prime}>1 / e$. For every $\epsilon>0$, such that $c-\epsilon>c^{\prime}$, we have

$$
\begin{aligned}
& \int_{h(\mathrm{t})}^{t} p(s) \\
& \quad \cdot \exp \left(\int_{\tau(s)}^{h(s)} p(u) \exp \left(\int_{\tau(u)}^{u} P_{j}(\xi, \epsilon) d \xi\right) d u\right) d s \\
& \quad>c-\epsilon>c^{\prime}>\frac{1}{e} .
\end{aligned}
$$

Combining inequalities (100) and (102), we obtain

$$
\ln \left(\frac{x(h(t))}{x(t)}\right)>c^{\prime}
$$

or

$$
\frac{x(h(t))}{x(t)}>e^{c^{\prime}}>e c^{\prime}>1,
$$

which yields

$$
x(h(t))>\left(e c^{\prime}\right) x(t) .
$$

where $P_{j}$ is defined by (38), then all solutions of $(E)$ oscillate. 
Following the above steps, we can inductively show that, for any positive integer $k$,

$$
\frac{x(h(t))}{x(t)}>\left(e c^{\prime}\right)^{k} \text { for sufficiently large } t \text {. }
$$

Since $e c^{\prime}>1$, there is a natural number $k \in \mathbb{N}$, satisfying $k>2\left[\ln 2-\ln c^{\prime}\right] /\left(1+\ln c^{\prime}\right)$ such that for $t$ sufficiently large

$$
\frac{x(h(t))}{x(t)}>\left(e c^{\prime}\right)^{k}>\left(\frac{2}{c^{\prime}}\right)^{2} .
$$

Further (cf. $[13,24])$, for sufficiently large $t$, there exists a real number $t_{m} \in(h(t), t)$, such that

$$
\begin{aligned}
& \int_{h(t)}^{t_{m}} p(s) \\
& \cdot \exp \left(\int_{\tau(s)}^{h(s)} p(u) \exp \left(\int_{\tau(u)}^{u} P_{j}(\xi, \epsilon) d \xi\right) d u\right) d s \\
& \quad>\frac{c^{\prime}}{2}, \\
& \int_{t_{m}}^{t} p(s) \exp \left(\int_{\tau(s)}^{h(s)} p(u) \exp \left(\int_{\tau(u)}^{u} P_{j}(\xi, \epsilon) d \xi\right) d u\right) d s \\
& \quad>\frac{c^{\prime}}{2} .
\end{aligned}
$$

Integrating (E) from $h(t)$ to $t_{m}$, using (85) and the fact that $x(t)>0$, we obtain

$$
\begin{aligned}
& x(h(t))>x\left(h\left(t_{m}\right)\right) \int_{h(t)}^{t_{m}} p(s) \\
& \quad \cdot \exp \left(\int_{\tau(s)}^{h(s)} p(u) \exp \left(\int_{\tau(u)}^{u} P_{j}(\xi, \epsilon) d \xi\right) d u\right) d s,
\end{aligned}
$$

which, in view of the first inequality in (108), implies that

$$
x(h(t))>\frac{c^{\prime}}{2} x\left(h\left(t_{m}\right)\right) .
$$

Similarly, integrating (E) from $t_{m}$ to $t$, using (85) and the fact that $x(t)>0$, we have

$$
\begin{aligned}
& x\left(t_{m}\right)>x(h(t)) \int_{t_{m}}^{t} p(s) \\
& \quad \cdot \exp \left(\int_{\tau(s)}^{h(s)} p(u) \exp \left(\int_{\tau(u)}^{u} P_{j}(\xi, \epsilon) d \xi\right) d u\right) d s,
\end{aligned}
$$

which, in view of the second inequality in (108), yields

$$
x\left(t_{m}\right)>\frac{c^{\prime}}{2} x(h(t)) .
$$

Combining inequalities (110) and (112), we deduce that

$$
x\left(h\left(t_{m}\right)\right)<\frac{2}{c^{\prime}} x(h(t))<\left(\frac{2}{c^{\prime}}\right)^{2} x\left(t_{m}\right),
$$

which contradicts (107).

The proof of the theorem is complete.
3.2. ADEs. Analogous oscillation conditions to those obtained for the delay equation (E) can be derived for the (dual) advanced differential equation $\left(\mathrm{E}^{\prime}\right)$ by following similar arguments with the ones employed for obtaining Theorems 6-10.

Theorem 11. Let $\rho(t)$ be defined by (27) and for some $j \in \mathbb{N}$

$$
\begin{aligned}
& \limsup _{t \rightarrow \infty} \int_{t}^{\rho(t)} q(s) \\
& \quad \cdot \exp \left(\int_{\rho(t)}^{\sigma(s)} q(u) \exp \left(\int_{u}^{\sigma(u)} Q_{j}(\xi) d \xi\right) d u\right) d s \\
& \quad>1,
\end{aligned}
$$

where

$$
\begin{aligned}
& Q_{j}(t)=q(t)\left[1+\int_{t}^{\sigma(t)} q(s)\right. \\
& \left.\quad \cdot \exp \left(\int_{t}^{\sigma(s)} q(u) \exp \left(\int_{u}^{\sigma(u)} Q_{j-1}(\xi) d \xi\right) d u\right) d s\right]
\end{aligned}
$$

with $Q_{0}(t)=\lambda_{0} q(t)$, and let $\lambda_{0}$ be the smaller root of the equation $\lambda=e^{\beta \lambda}$. Then all solutions of $\left(E^{\prime}\right)$ oscillate.

Theorem 12. Let $\rho(t)$ be defined by (27) and $\beta \in(0,1 / e]$. If for some $j \in \mathbb{N}$

$$
\begin{aligned}
& \limsup _{t \rightarrow \infty} \int_{t}^{\rho(t)} q(s) \\
& \quad \cdot \exp \left(\int_{\rho(t)}^{\sigma(s)} q(u) \exp \left(\int_{u}^{\sigma(u)} Q_{j}(\xi) d \xi\right) d u\right) d s \\
& \quad>1-D(\beta)
\end{aligned}
$$

where $Q_{j}$ is defined by (115), then all solutions of $\left(E^{\prime}\right)$ oscillate.

Theorem 13. Let $\rho(t)$ be defined by (27) and $\beta \in(0,1 / e]$. If for some $j \in \mathbb{N}$

$$
\begin{aligned}
& \underset{t \rightarrow \infty}{\limsup } \int_{t}^{\rho(t)} q(s) \\
& \quad \exp \left(\int_{t}^{\sigma(s)} q(u) \exp \left(\int_{u}^{\sigma(u)} Q_{j}(\xi) d \xi\right) d u\right) d s \\
& >\frac{1}{D(\beta)},
\end{aligned}
$$

where $Q_{j}$ is defined by (115), then all solutions of $\left(E^{\prime}\right)$ oscillate. 
Theorem 14. Let $\rho(t)$ be defined by (27) and $\beta \in(0,1 / e]$. If for some $j \in \mathbb{N}$

$$
\begin{aligned}
& \limsup _{t \rightarrow \infty} \int_{t}^{\rho(t)} q(s) \\
& \cdot \exp \left(\int_{\rho(s)}^{\sigma(s)} q(u) \exp \left(\int_{u}^{\sigma(u)} Q_{j}(\xi) d \xi\right) d u\right) d s \\
& >\frac{1+\ln \lambda_{0}}{\lambda_{0}}-D(\beta),
\end{aligned}
$$

where $Q_{j}$ is defined by (115) and $\lambda_{0}$ is the smaller root of the equation $\lambda=e^{\beta \lambda}$, then all solutions of $\left(E^{\prime}\right)$ oscillate.

Theorem 15. Let $\rho(t)$ be defined by (27). If for some $j \in \mathbb{N}$

$$
\begin{aligned}
& \liminf _{t \rightarrow \infty} \int_{t}^{\rho(t)} q(s) \\
& \cdot \exp \left(\int_{\rho(s)}^{\sigma(s)} q(u) \exp \left(\int_{u}^{\sigma(u)} Q_{j}(\xi) d \xi\right) d u\right) d s \\
& \quad>\frac{1}{e},
\end{aligned}
$$

where $Q_{j}$ is defined by (115), then all solutions of $\left(E^{\prime}\right)$ oscillate.

3.3. Differential Inequalities. A slight modification in the proofs of Theorems 6-15 leads to the following results about differential inequalities.

Theorem 16. Assume that all the conditions of Theorem 6 [11], 7 [12], 8 [13], 9 [14], or 10 [15] hold. Then

(i) the delay [advanced] differential inequality

$$
\begin{aligned}
& x^{\prime}(t)+p(t) x(\tau(t)) \leq 0 \\
& {\left[x^{\prime}(t)-q(t) x(\sigma(t)) \geq 0\right],} \\
& \quad t \geq t_{0}
\end{aligned}
$$

has no eventually positive solutions;

(ii) the delay [advanced] differential inequality

$$
\begin{gathered}
x^{\prime}(t)+p(t) x(\tau(t)) \geq 0 \\
{\left[x^{\prime}(t)-q(t) x(\sigma(t)) \leq 0\right],}
\end{gathered}
$$

has no eventually negative solutions.

Remark 17. The oscillation criteria established in this paper all depend on $\lambda_{0}$ (see, e.g., (37) and (71)) in contrast to the conditions obtained in $[15,16]$ and in $[17$, for $m=1]$. In fact, the left-hand side of conditions (37) and (71) depends on $\lambda_{0}$, which is not the case with the left-hand side of conditions (20) and (21). Since $\lambda_{0}>1$ when $\alpha \in(0,1 / e]$, it is obvious that

$$
P_{0}(t)=\lambda_{0} p(t)>p(t)=p_{0}(t) .
$$

Consequently, the left-hand side of conditions (37) and (71) is greater than the corresponding parts of (20) and (21), respectively. This is the reason why the conditions in this paper improve on all known conditions mentioned in Section 2.

\section{Examples and Comments}

The oscillation tests we have proposed and established, in the main results, involve an iterative procedure. We iteratively compute limsup and liminf on the terms $P_{j}(t)$ and $Q_{j}(t), j \in$ $\mathbb{N}$ of a recurrent relation defined on the coefficients and the deviating argument of an equation of the form (E) or $\left(E^{\prime}\right)$ to determine whether that equation is oscillatory. But this computation cannot be performed on paper, but by means of a program, numerically computing limsup and liminf. The examples below illustrate the significance of our results and indicate the high level of improvement in the oscillation criteria. The calculations were performed using MATLAB code.

Example 1. Consider the delay differential equation

$$
x^{\prime}(t)+\frac{3}{25} x(\tau(t))=0, \quad t \geq 0,
$$

with (see Figure 1(a))

$$
\tau(t)= \begin{cases}t-1, & \text { if } t \in[8 k, 8 k+2] \\ -4 t+40 k+9, & \text { if } t \in[8 k+2,8 k+3] \\ 5 t-32 k-18, & \text { if } t \in[8 k+3,8 k+4] \\ -4 t+40 k+18, & \text { if } t \in[8 k+4,8 k+5] \\ 5 t-32 k-27, & \text { if } t \in[8 k+5,8 k+6] \\ -2 t+24 k+15, & \text { if } t \in[8 k+6,8 k+7] \\ 6 t-40 k-41, & \text { if } t \in[8 k+7,8 k+8],\end{cases}
$$

where $k \in \mathbb{N}_{0}$ and $\mathbb{N}_{0}$ is the set of nonnegative integers.

By (13), we see (Figure 1(b)) that

$$
h(t)= \begin{cases}t-1, & \text { if } t \in[8 k, 8 k+2] \\ 8 k+1, & \text { if } t \in\left[8 k+2,8 k+\frac{19}{5}\right] \\ 5 t-32 k-18, & \text { if } t \in\left[8 k+\frac{19}{5}, 8 k+4\right] \\ 8 k+2, & \text { if } t \in\left[8 k+4,8 k+\frac{29}{5}\right] \\ 5 t-32 k-27, & \text { if } t \in\left[8 k+\frac{29}{5}, 8 k+6\right] \\ 8 k+3, & \text { if } t \in\left[8 k+6,8 k+\frac{44}{6}\right] \\ 6 t-40 k-41, & \text { if } t \in\left[8 k+\frac{44}{6}, 8 k+8\right] .\end{cases}
$$

It is obvious that

$$
\begin{aligned}
\alpha & =\liminf _{t \rightarrow \infty} \int_{\tau(t)}^{t} p(s) d s=\liminf _{t \rightarrow \infty} \int_{8 k+1}^{8 k+2} \frac{3}{25} d s \\
& =0.12
\end{aligned}
$$




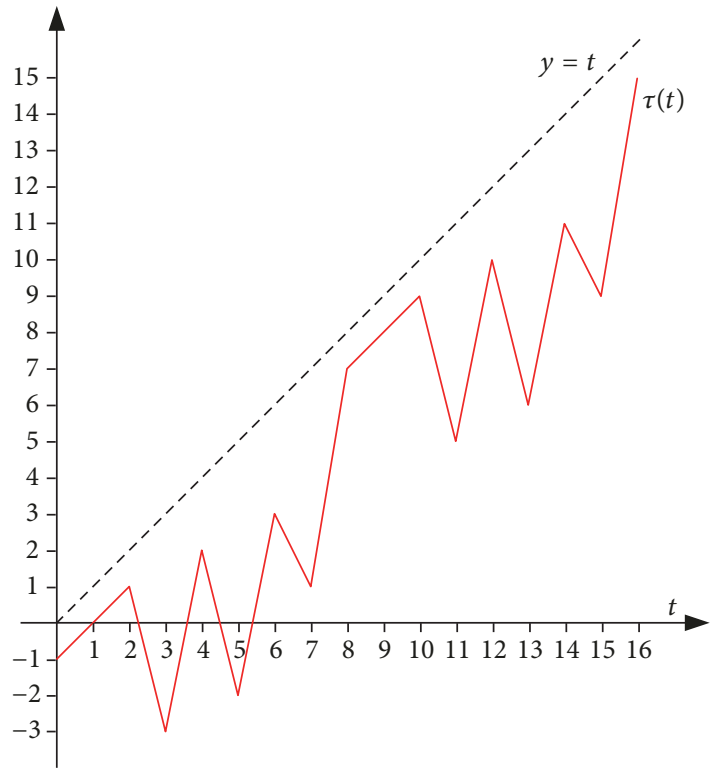

(a)

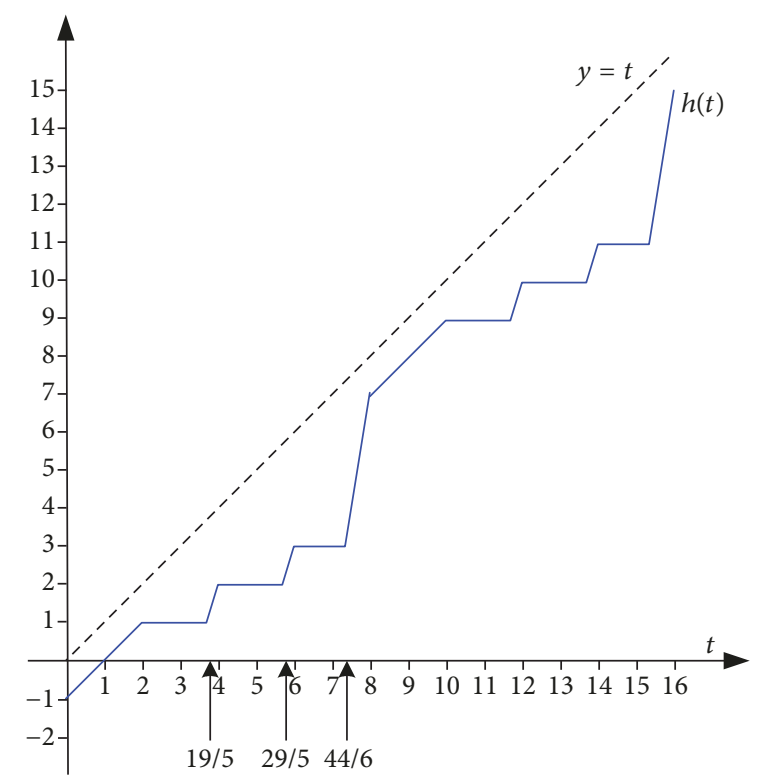

(b)

FIGURE 1: The graphs of $\tau(t)$ and $h(t)$.

and therefore, the smaller root of $e^{0.12 \lambda}=\lambda$ is $\lambda_{0}=1.14765$.

Observe that the function $F_{j}: \mathbb{R}_{0} \rightarrow \mathbb{R}_{+}$defined as

$$
\begin{aligned}
F_{j}(t) & =\int_{h(t)}^{t} p(s) \\
& \cdot \exp \left(\int_{\tau(s)}^{h(t)} p(u) \exp \left(\int_{\tau(u)}^{u} P_{j}(\xi) d \xi\right) d u\right) d s
\end{aligned}
$$

attains its maximum at $t=8 k+44 / 6, k \in \mathbb{N}_{0}$, for every $j \in \mathbb{N}$. Specifically,

$$
\begin{aligned}
& F_{1}\left(t=8 k+\frac{44}{6}\right)=\int_{8 k+3}^{8 k+44 / 6} p(s) \\
& \quad \cdot \exp \left(\int_{\tau(s)}^{8 k+3} p(u) \exp \left(\int_{\tau(u)}^{u} P_{1}(\xi) d \xi\right) d u\right) d s
\end{aligned}
$$

with

$$
\begin{aligned}
& P_{1}(\xi)=p(\xi)\left[1+\int_{\tau(\xi)}^{\xi} p(v)\right. \\
& \left.\quad \cdot \exp \left(\int_{\tau(v)}^{\xi} p(w) \exp \left(\int_{\tau(w)}^{w} \lambda_{0} p(z) d z\right) d w\right) d v\right] .
\end{aligned}
$$

Using MATLAB, we obtain

$$
F_{1}\left(t=8 k+\frac{44}{6}\right) \simeq 1.0417
$$

and therefore

$$
\limsup _{t \rightarrow \infty} F_{1}(t) \simeq 1.0417>1 .
$$

Hence, condition (37) of Theorem 6 is satisfied, for $j=1$. Consequently, all solutions of (123) are oscillatory.

Observe, however, that

$$
\begin{aligned}
\mathrm{LD} & =\limsup _{k \rightarrow \infty} \int_{8 k+3}^{8 k+44 / 6} \frac{3}{35} d s=0.52<1, \\
\alpha & =0.12<\frac{1}{e} \\
0.52 & <\frac{1+\ln \lambda_{0}}{\lambda_{0}}-D(\alpha) \simeq 0.9831 .
\end{aligned}
$$

Note that the function $\Phi_{j}$ defined by

$$
\Phi_{j}(t)=\int_{h(t)}^{t} p(s) \exp \left(\int_{h(s)}^{h(t)} p(u) \psi_{j}(u) d u\right) d s,
$$

$$
j \geq 2 \text {, }
$$

attains its maximum at $t=8 k+44 / 6, k \in \mathbb{N}_{0}$, for every $j \geq 2$. Specifically,

$$
\begin{aligned}
& \Phi_{2}\left(8 k+\frac{44}{6}\right) \\
& =\int_{8 k+3}^{8 k+44 / 6} p(s) \exp \left(\int_{h(s)}^{8 k+3} p(s) \psi_{2}(u) d u\right) d s \\
& =\int_{8 k+3}^{8 k+44 / 6} \frac{3}{25} \exp \left(\int_{h(s)}^{8 k+3} \frac{3}{25} \exp \left(\int_{\tau(u)}^{u} \frac{3}{25} \cdot 0 d w\right) d u\right) d s \\
& =\int_{8 k+3}^{8 k+44 / 6} \frac{3}{25} \exp \left(\int_{h(s)}^{8 k+3} \frac{3}{25} \cdot 1 d u\right) d s \\
& =\frac{3}{25} \cdot\left[\int_{8 k+3}^{8 k+19 / 5} \exp \left(\frac{3}{25} \int_{8 k+1}^{8 k+3} d u\right) d s\right.
\end{aligned}
$$




$$
\begin{aligned}
& +\int_{8 k+19 / 5}^{8 k+4} \exp \left(\frac{3}{25} \int_{5 s-32 k-18}^{8 k+3} d u\right) d s \\
& +\int_{8 k+4}^{8 k+29 / 5} \exp \left(\frac{3}{25} \int_{8 k+2}^{8 k+3} d u\right) d s \\
& +\int_{8 k+29 / 5}^{8 k+6} \exp \left(\frac{3}{25} \int_{5 s-32 k-27}^{8 k+3} d u\right) d s \\
& \left.+\int_{8 k+6}^{8 k+44 / 6} \exp \left(\frac{3}{25} \int_{8 k+3}^{8 k+3} d u\right) d s\right] \simeq 0.57983
\end{aligned}
$$

Thus

$$
\limsup _{t \rightarrow \infty} \Phi_{2}(t) \simeq 0.57983<1-D(\alpha) \simeq 0.99174 .
$$

Also

$$
\begin{aligned}
& \limsup _{t \rightarrow \infty} \int_{h(t)}^{t} p(s) \exp \left(\int_{\tau(s)}^{h(t)} p(u) d u\right) d s \\
& \quad=\limsup _{k \rightarrow \infty} \int_{8 k+3}^{8 k+44 / 6} \frac{3}{25} \exp \left(\int_{\tau(s)}^{8 k+3} \frac{3}{25} d u\right) d s \\
& \quad=\frac{3}{25} \cdot \limsup _{t \rightarrow \infty}\left[\int_{8 k+3}^{8 k+4} \exp \left(\frac{3}{25} \int_{5 s-32 k-18}^{8 k+3} d u\right) d s\right. \\
& \quad+\int_{8 k+4}^{8 k+5} \exp \left(\frac{3}{25} \int_{-4 s+40 k+18}^{8 k+3} d u\right) d s \\
& \quad+\int_{8 k+5}^{8 k+6} \exp \left(\frac{3}{25} \int_{5 s-32 k-27}^{8 k+3} d u\right) d s \\
& \quad+\int_{8 k+6}^{8 k+7} \exp \left(\frac{3}{25} \int_{-2 s+24 k+15}^{8 k+3} d u\right) d s \\
& \left.\quad+\int_{8 k+7}^{8 k+44 / 6} \exp \left(\frac{3}{25} \int_{6 s-40 k-41}^{8 k+3} d u\right) d s\right] \simeq 0.7043 \\
& \quad<1, \\
& 0.7043<1-D(\alpha) \simeq 0.99174 .
\end{aligned}
$$

In addition,

$$
\begin{aligned}
& \limsup _{t \rightarrow \infty} \int_{h(t)}^{t} p(s) \exp \left(\int_{\tau(s)}^{h(t)} p_{1}(u) d u\right) d s \simeq 0.8052 \\
& \quad<1, \\
& 0.8052<1-D(\alpha) \simeq 0.99174 .
\end{aligned}
$$

That is, none of conditions (8), (9), (12), (14) (for $j=2)$, (16), (17), (20) (for $j=1$ ), and (21) (for $j=1$ ) is satisfied.

Comment. The improvement of condition (37) over the corresponding condition (8) is significant, approximately $100.33 \%$. We get this measure by comparing the values, in the left-hand side of those conditions. Also, the improvement over conditions (14), (16), and (20) is very satisfactory, around $79.66 \%$, $47.9 \%$, and $29.37 \%$, respectively. In addition, condition (37) is satisfied from the first iteration, while conditions (14), (20), and (21) need more than one iteration.

Example 2 (taken and adapted from [17]). Consider the advanced differential equation

$$
x^{\prime}(t)-\frac{333}{2500} x(\sigma(t))=0, \quad t \geq 0,
$$

with (see Figure 2(a))

$$
\sigma(t)= \begin{cases}5 k+3, & \text { if } t \in[5 k, 5 k+1] \\ 4 t-15 k-1, & \text { if } t \in[5 k+1,5 k+2] \\ -3 t+20 k+13, & \text { if } t \in[5 k+2,5 k+3] \\ 5 t-20 k-11, & \text { if } t \in[5 k+3,5 k+4] \\ -t+10 k+13, & \text { if } t \in[5 k+4,5 k+5],\end{cases}
$$

where $k \in \mathbb{N}_{0}$ and $\mathbb{N}_{0}$ is the set of nonnegative integers.

By (27), we see (Figure 2(b)) that

$$
\rho(t)= \begin{cases}5 k+3, & \text { if } t \in[5 k, 5 k+1] \\ 4 t-15 k-1, & \text { if } t \in[5 k+1,5 k+1.25] \\ 5 k+4, & \text { if } t \in[5 k+1.25,5 k+3] \\ 5 t-20 k-11, & \text { if } t \in[5 k+3,5 k+3.8] \\ 5 k+8, & \text { if } t \in[5 k+3.8,5 k+5] .\end{cases}
$$

It is obvious that

$$
\beta=\liminf _{t \rightarrow \infty} \int_{5 k+3}^{5 k+4} \frac{333}{2500} d s=0.1332
$$

and therefore, the smaller root of $e^{0.1332 \lambda}=\lambda$ is $\lambda_{0}=1.16839$.

Observe, that the function $G_{j}: \mathbb{R}_{0} \rightarrow \mathbb{R}_{+}$defined as

$$
\begin{aligned}
& G_{j}(t)=\int_{t}^{\rho(t)} q(s) \\
& \cdot \exp \left(\int_{\rho(t)}^{\sigma(s)} q(u) \exp \left(\int_{u}^{\sigma(u)} Q_{j}(\xi) d \xi\right) d u\right) d s
\end{aligned}
$$

attains its maximum at $t=5 k+3.8, k \in \mathbb{N}_{0}$, for every $j \in \mathbb{N}$. Specifically,

$$
\begin{aligned}
& G_{1}(t=5 k+3.8)=\int_{5 k+3.8}^{5 k+8} q(s) \\
& \quad \cdot \exp \left(\int_{5 k+8}^{\sigma(s)} q(u) \exp \left(\int_{u}^{\sigma(u)} Q_{1}(\xi) d \xi\right) d u\right) d s
\end{aligned}
$$

with

$$
\begin{aligned}
& Q_{1}(\xi)=q(\xi)\left[1+\int_{\xi}^{\sigma(\xi)} q(v)\right. \\
& \left.\quad \cdot \exp \left(\int_{\xi}^{\sigma(v)} q(w) \exp \left(\int_{w}^{\sigma(w)} \lambda_{0} q(z) d z\right) d w\right) d v\right] .
\end{aligned}
$$




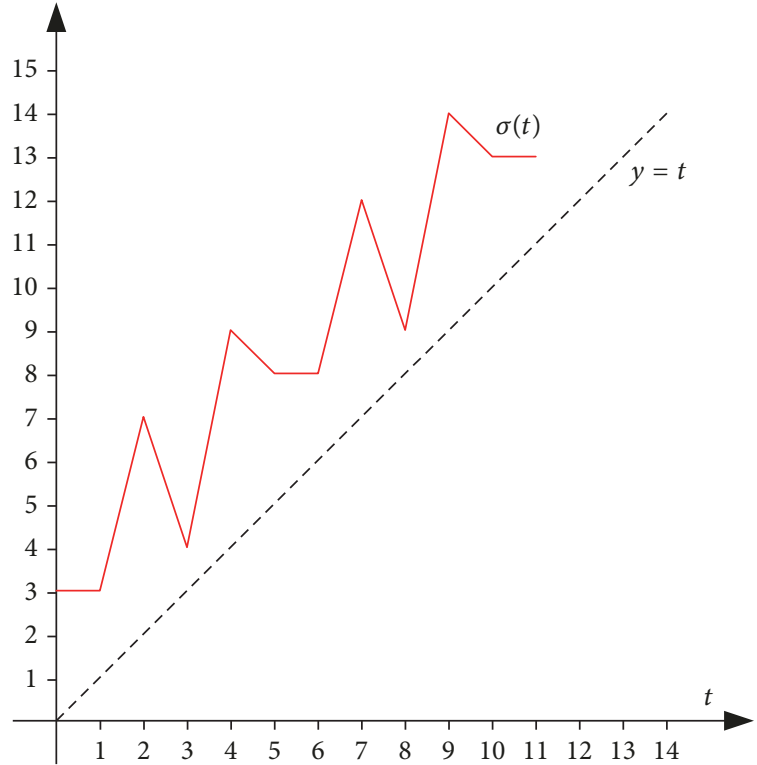

(a)

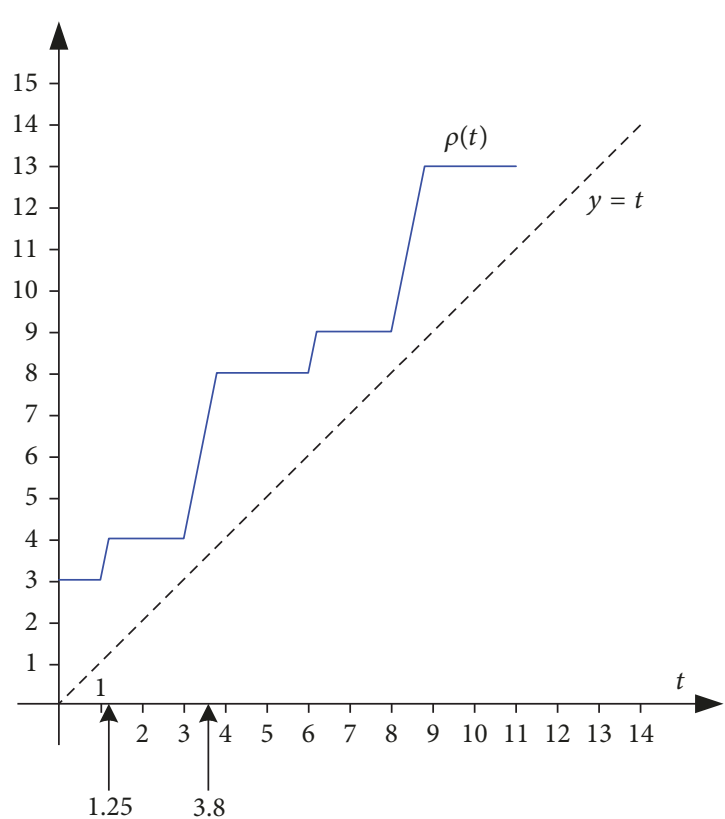

(b)

Figure 2: The graphs of $\sigma(t)$ and $\rho(t)$.

Using MATLAB, we obtain

$$
G_{1}(t=5 k+3.8) \simeq 0.9915 \text {. }
$$

Therefore

$$
\limsup _{t \rightarrow \infty} G_{1}(t) \simeq 0.9915>1-D(\beta) \simeq 0.9896 .
$$

Hence, condition (116) of Theorem 12 is satisfied, for $j=1$. Consequently, all solutions of (138) oscillate.

Observe, however, that

$$
\begin{aligned}
& \text { LA }=\limsup _{k \rightarrow \infty} \int_{5 k+3.8}^{5 k+8} \frac{333}{2500} d s=0.55944<1, \\
& \beta=0.1332<\frac{1}{e}, \\
& \limsup _{t \rightarrow \infty} \int_{t}^{\rho(t)} q(s) \exp \left(\int_{\rho(t)}^{\sigma(s)} q(u) d u\right) d s \\
&=\limsup _{k \rightarrow \infty} \int_{5 k+3.8}^{5 k+8} q(s) \exp \left(\int_{5 k+8}^{\sigma(s)} q(u) d u\right) d s \\
&=\limsup _{k \rightarrow \infty}\left[\int_{5 k+3.8}^{5 k+4} q(s)\right. \\
& \quad \cdot \exp \left(\int_{5 k+8}^{5 s-20 k-11} q(u) d u\right) d s+\int_{5 k+4}^{5 k+5} q(s) \\
& \quad \cdot \exp \left(\int_{5 k+8}^{-s+10 k+13} q(u) d u\right) d s+\int_{5 k+5}^{5 k+6} q(s) \\
& \quad \cdot \exp \left(\int_{5 k+8}^{5 k+8} q(u) d u\right) d s+\int_{5 k+6}^{5 k+7} q(s)
\end{aligned}
$$

$$
\begin{aligned}
& \cdot \exp \left(\int_{5 k+8}^{4 s-15 k-16} q(u) d u\right) d s+\int_{5 k+7}^{5 k+8} q(s) \\
& \left.\cdot \exp \left(\int_{5 k+8}^{-3 s+20 k+33} q(u) d u\right) d s\right] \simeq 0.6672<1 \\
& \liminf _{t \rightarrow \infty} \int_{t}^{\rho(t)} q(s) \exp \left(\int_{\rho(t)}^{\sigma(s)} q(u) d u\right) d s \\
& \quad=\liminf _{t \rightarrow \infty} \int_{5 k+3}^{5 k+4} q(s) \exp \left(\int_{5 k+4}^{\sigma(s)} q(u) d u\right) d s \\
& \left.\quad \simeq 0.1893<\frac{1}{e}, \quad \int^{\rho} q_{1}(u) d u\right) d s \simeq 0.7196 \\
& \quad<1, \\
& \limsup _{t \rightarrow \infty} \int_{t}^{\rho(t)} q(s) \exp \left(\int_{\rho(t)}^{\sigma(s)}{ }^{0.7196<1-D(\beta) \simeq 0.9896 .}\right.
\end{aligned}
$$

That is, none of conditions (24), (25), (28), (29), (30) (for $j=$ 1 ), and (31) (for $j=1$ ) is satisfied.

Comment. The improvement of condition (116) over the corresponding condition (24) is significant, approximately $77.23 \%$. We get this measure by comparing the values, in the left-hand side of those conditions. Also, the improvement over conditions (28) and (30) is very satisfactory, around $48.61 \%$ and $37.78 \%$, respectively. In addition, condition (116) is satisfied from the first iteration, while conditions (30) and (31) need more than one iteration. 
Remark 3. Similarly, one can provide examples, illustrating the other main results.

\section{Concluding Remarks}

In the present paper, we have considered the oscillatory dynamics of differential equations, having nonmonotone deviating arguments and nonnegative coefficients. New sufficient conditions have been established, for the oscillation of all solutions of $(\mathrm{E})$ and $\left(\mathrm{E}^{\prime}\right)$. These conditions include (37), (71), (75), (83), and (97) and (114), (116), (117), (118), and (119), for (E) and ( $\left.E^{\prime}\right)$, respectively. Applying these conditions involves a procedure that checks for oscillations by iteratively computing limsup and liminf, on terms recursively defined on the equation's coefficients and deviating argument.

The main advantage of these conditions is that they achieve a major improvement over all the related oscillation conditions for $(E)\left[\left(E^{\prime}\right)\right]$, in the literature. For example, condition (37) [(114)] improves upon the noniterative conditions that are reviewed in the introduction, namely, conditions (8)

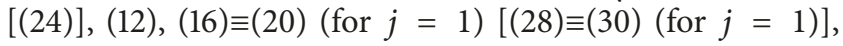
and (17) $\equiv(21)$ (for $j=1)$ [(31) (for $j=1)$ ]. That immediately becomes evident by inspecting the left-hand side of (37) [(114)] and the left-hand side of each of the above conditions.

The improvement of (37) [(114)] over the other iterative conditions, namely, (14) (for $j>2),(20)($ for $j>1)$ [(30) (for $j>1)$ ], and (21) (for $j>1$ ) [(31) (for $j>1)$ ], is that it requires far fewer iterations to establish oscillation than the other conditions.

This advantage, easily, can be verified computationally, by running the MATLAB programs (see Appendix), for computing limsup and liminf and comparing the number of iterations required by each condition to establish oscillation. Then we see that we achieve a significant improvement over all known oscillation criteria.

Another advantage and a significant departure from the large majority of the other studies is that the criteria in this paper apply to a more general class of equations, having nonmonotone arguments $\tau(t)$ or $\sigma(t)$, in contrast to most of the other oscillation criteria that apply to equations with nondecreasing arguments.

\section{Appendix}

In this appendix, for completeness, we give the algorithm on MATLAB software used in Example 1 for calculation of $\lim \sup _{t \rightarrow \infty} F_{1}(t) \simeq 1.0417$. For Example 2, the algorithm is omitted since it is similar to the one in Example 1.

Algorithm for Example 1

clear; clc;

$\mathrm{c}=.12$;

$n=50$

$\lambda 0=1.14765 ;$

a5 $=19$;

b5 = 70/3;

h5 $=(\mathrm{b} 5-\mathrm{a} 5) / n$;

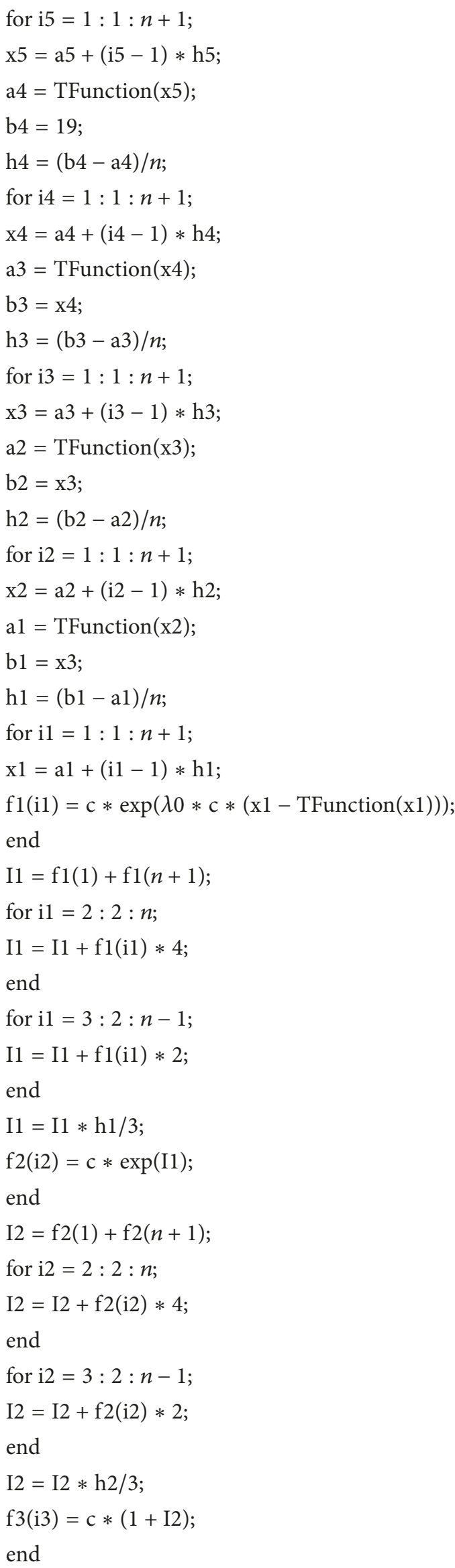




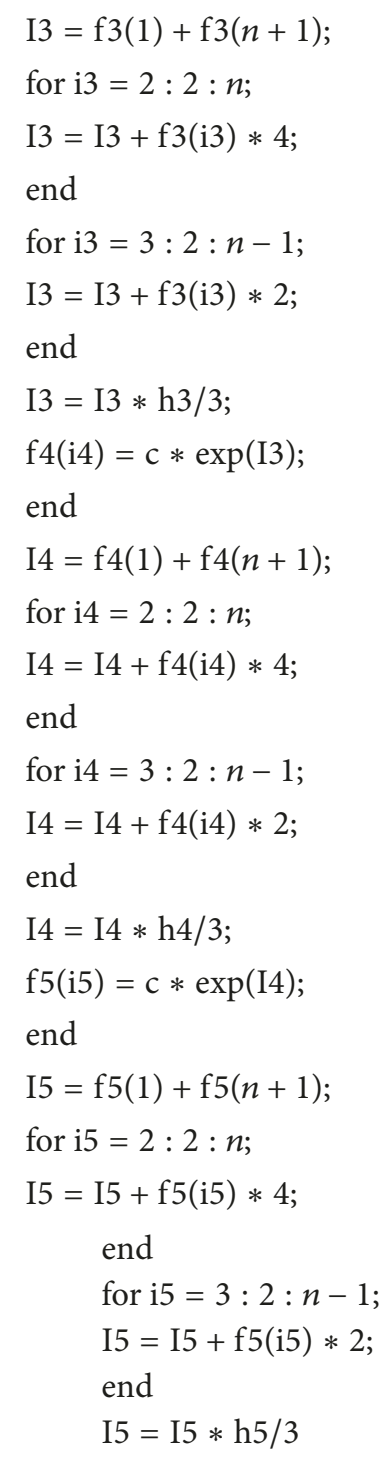

Algorithms for functions $\tau(t)$ and $h(t)$

function $[\mathrm{a}]=$ TFunction $(\mathrm{x})$

$r=\bmod (x, 8)$;

$\mathrm{k}=$ floor $(x / 8)$;

if $(r>=0) \& \&(r<2)$

$\mathrm{a}=\mathrm{x}-1$;

end

if $(r>=2) \& \&(r<3)$

$\mathrm{a}=-4 * \mathrm{x}+40 * \mathrm{k}+9$;

end

if $(r>=3) \& \&(r<4)$

$\mathrm{a}=5 * \mathrm{x}-32 * \mathrm{k}-18$;

end

if $(r>=4) \& \&(r<5)$

$\mathrm{a}=-4 * \mathrm{x}+40 * \mathrm{k}+18$;

end if $(r>=5) \& \&(r<6)$

$\mathrm{a}=5 * \mathrm{x}-32 * \mathrm{k}-27$;

end

if $(r>=6) \& \&(r<7)$

$\mathrm{a}=-2 * \mathrm{x}+24 * \mathrm{k}+15$;

end

if $(r>=7) \& \&(r<8)$

$\mathrm{a}=6 * \mathrm{x}-40 * \mathrm{k}-41$;

end

end

function $[\mathrm{a}]=\operatorname{HFunction}(\mathrm{x})$

$r=\bmod (x, 8)$;

$\mathrm{k}=$ floor $(x / 8)$;

if $(r>=0) \quad \& \&(r<2)$

$\mathrm{a}=\mathrm{x}-1$;

end

if $(r>=2) \& \&(r<19 / 5)$

$\mathrm{a}=8 * \mathrm{k}+1$;

end

if $(r>=19 / 5) \quad \& \&(r<4)$

$\mathrm{a}=5 * \mathrm{x}-32 * \mathrm{k}-18$;

end

if $(r>=4) \& \&(r<29 / 5)$

$\mathrm{a}=8 * \mathrm{k}+2$;

end

if $(r>=29 / 5) \& \&(r<6)$

$\mathrm{a}=5 * \mathrm{x}-32 * \mathrm{k}-27$;

end

if $(r>=6) \& \&(r<44 / 6)$

$\mathrm{a}=8 * \mathrm{k}+3$;

end

if $(r>=44 / 6) \& \&(r<8)$

$\mathrm{a}=6 * \mathrm{x}-40 * \mathrm{k}-41$;

end

end

\section{Conflicts of Interest}

The authors declare that they have no conflicts of interest. 


\section{Acknowledgments}

The research of the second author is supported by the National Natural Science Foundation of China (Grant no. 61503171), China Postdoctoral Science Foundation (Grant no. 2015M582091), Natural Science Foundation of Shandong Province (Grant no. ZR2016JL021), Doctoral Scientific Research Foundation of Linyi University (Grant no. LYDX2015BS001), and the Applied Mathematics Enhancement Program of Linyi University.

\section{References}

[1] M. Bani-Yaghoub, "Analysis and applications of delay differential equations in biology and medicine," https://arxiv.org/abs/ 1701.04173 .

[2] G. A. Bocharov and F. A. Rihan, "Numerical modelling in biosciences using delay differential equations," Journal of Computational and Applied Mathematics, vol. 125, no. 1-2, pp. 183199, 2000.

[3] F. Brauer and C. Castillo-Chávez, Mathematical Models in Population Biology and Epidemiology, vol. 40 of Texts in Applied Mathematics, Springer, New York, NY, USA, 2nd edition, 2012.

[4] U. Foryś, "Marchuk's model of immune system dynamics with application to tumour growth," Journal of Theoretical Medicine, vol. 4, no. 1, pp. 85-93, 2002.

[5] K. Gopalsamy, Stability and Oscillations in Delay Differential Equations of Population Dynamics, vol. 74 of Mathematics and Its Applications, Kluwer Academic Publishers, Dordrecht, The Netherlands, 1992.

[6] G. E. Hutchinson, "Circular causal systems in ecology," Annals of the New York Academy of Sciences, vol. 50, no. 4, pp. 221-246, 1948.

[7] F. A. Rihan and M. N. Anwar, "Qualitative analysis of delayed SIR epidemic model with a saturated incidence rate," International Journal of Differential Equations, vol. 2012, Article ID 408637, 13 pages, 2012.

[8] F. A. Rihan, D. H. Abdel Rahman, S. Lakshmanan, and A. S. Alkhajeh, "A time delay model of tumour-immune system interactions: global dynamics, parameter estimation, sensitivity analysis," Applied Mathematics and Computation, vol. 232, pp. 606-623, 2014.

[9] F. A. Rihan and B. F. Rihan, "Numerical modelling of biological systems with memory using delay differential equations," Applied Mathematics \& Information Sciences, vol. 9, no. 3, pp. 1645-1658, 2015.

[10] H. Smith, An Introduction to Delay Differential Equations with Applications to the Life Sciences, Springer, New York, NY, USA, 2011.

[11] R. D. Driver, "Can the future influence the present?" Physical Review D: Particles, Fields, Gravitation and Cosmology, vol. 19, no. 4, pp. 1098-1107, 1979.

[12] L. E. El'sgol'ts and S. B. Norkin, Introduction to the Theory and Application of Differential Equations with Deviating Arguments, Academic Press, New York, NY, USA, 1973.

[13] E. Braverman, G. E. Chatzarakis, and I. P. Stavroulakis, "Iterative oscillation tests for differential equations with several nonmonotone arguments," Advances in Difference Equations, vol. 2016, Article ID 87, 18 pages, 2016.

[14] E. Braverman and B. Karpuz, "On oscillation of differential and difference equations with non-monotone delays," Applied
Mathematics and Computation, vol. 218, no. 7, pp. 3880-3887, 2011.

[15] G. E. Chatzarakis, "Differential equations with non-monotone arguments: iterative oscillation results," Journal of Mathematical and Computational Science, vol. 6, no. 5, pp. 953-964, 2016.

[16] G. E. Chatzarakis, "On oscillation of differential equations with non-monotone deviating arguments," Mediterranean Journal of Mathematics, vol. 14, no. 2, Article ID 82, 17 pages, 2017.

[17] G. E. Chatzarakis, "Oscillations caused by several nonmonotone deviating arguments," Differential Equations \& Applications, vol. 9, no. 3, pp. 285-310, 2017.

[18] G. E. Chatzarakis and Ö. Öcalan, "Oscillations of differential equations with several non-monotone advanced arguments," Dynamical Systems, vol. 30, no. 3, pp. 310-323, 2015.

[19] L. H. Erbe, Q. Kong, and B. G. Zhang, Oscillation Theory for Functional Differential Equations, Marcel Dekker, New York, NY, USA, 1995.

[20] L. H. Erbe and B. G. Zhang, "Oscillation for first order linear differential equations with deviating arguments," Differential and Integral Equations: International Journal for Theory and Applications, vol. 1, no. 3, pp. 305-314, 1988.

[21] N. Fukagai and T. Kusano, "Oscillation theory of first order functional differential equations with deviating arguments," Annali di Matematica Pura ed Applicata. Serie Quarta, vol. 136, no. 1, pp. 95-117, 1984.

[22] I. Győri and G. Ladas, Oscillation Theory of Delay Differential Equations, Oxford Mathematical Monographs, Clarendon Press, New York, NY, USA, 1991.

[23] J. Jaroš and I. P. Stavroulakis, "Oscillation tests for delay equations," Rocky Mountain Journal of Mathematics, vol. 29, no. 1, pp. 197-207, 1999.

[24] R. G. Koplatadze and T. A. Chanturiya, "Oscillating and monotone solutions of first-order differential equations with deviating argument," Differentsial'nye Uravneniya, vol. 18, no. 8, pp. 1463-1465, 1472, 1982.

[25] R. Koplatadze and G. Kvinikadze, "On the oscillation of solutions of first order delay differential inequalities and equations," Georgian Mathematical Journal, vol. 1, no. 6, pp. 675-685, 1994.

[26] M. K. Kwong, "Oscillation of first-order delay equations," Journal of Mathematical Analysis and Applications, vol. 156, no. 1, pp. 274-286, 1991.

[27] G. Ladas, V. Lakshmikantham, and J. S. Papadakis, "Oscillations of higher-order retarded differential equations generated by the retarded argument," Delay and functional differential equations and their applications (Proc. Conf., Park City, Utah, 1972), pp. 219-231, 1972.

[28] G. S. Ladde, "Oscillations caused by retarded perturbations of first order linear ordinary differential equations," Atti della Accademia Nazionale dei Lincei. Rendiconti della Classe di Scienze Fisiche, Matematiche e Naturali, vol. 63, no. 5, pp. 351359 (1978), 1977.

[29] G. S. Ladde, V. Lakshmikantham, and B. G. Zhang, Oscillation Theory of Differential Equations with Deviating Arguments, vol. 110 of Monographs and Textbooks in Pure and Applied Mathematics, Marcel Dekker, New York, NY, USA, 1987.

[30] H. A. El-Morshedy and E. R. Attia, "New oscillation criterion for delay differential equations with non-monotone arguments," Applied Mathematics Letters, vol. 54, pp. 54-59, 2016.

[31] A. D. Myškis, "Linear homogeneous differential equations of the first order with retarded argument," Akademiya Nauk SSSR $i$ Moskovskoe Matematicheskoe Obshchestvo Uspekhi Matematicheskikh Nauk, vol. 5, no. 2(36), pp. 160-162, 1950. 
[32] I. P. Stavroulakis, "Oscillation criteria for delay and difference equations with non-monotone arguments," Applied Mathematics and Computation, vol. 226, pp. 661-672, 2014.

[33] D. Zhou, "On some problems on oscillation of functional differential equations of first order," Journal of Shandong University (Natural Science), vol. 25, no. 4, pp. 434-442, 1990. 


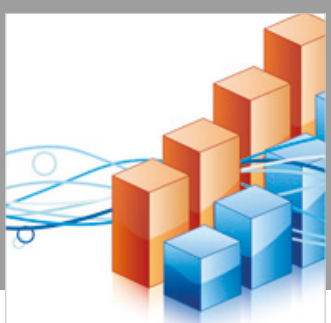

Advances in

Operations Research

\section{-n-m}
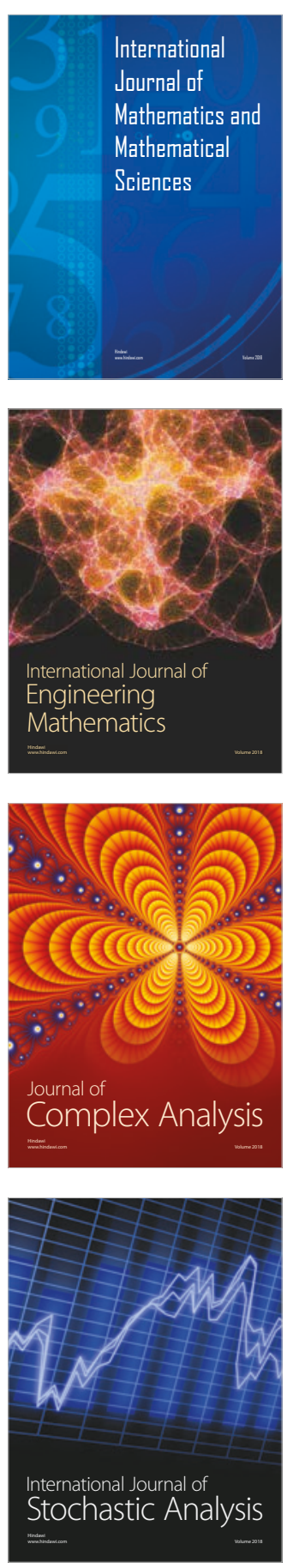
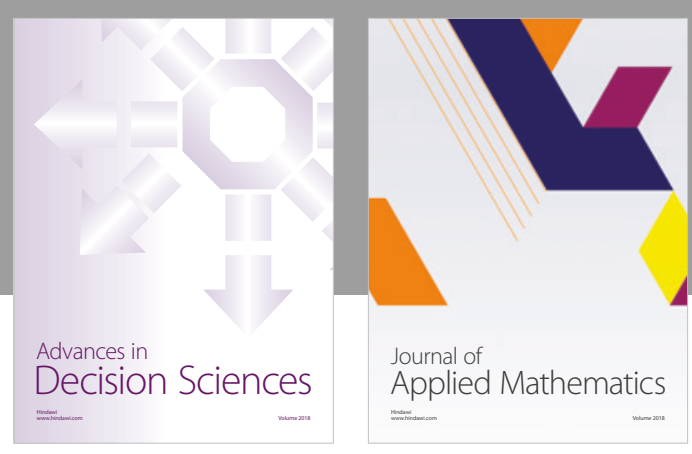

Journal of

Applied Mathematics
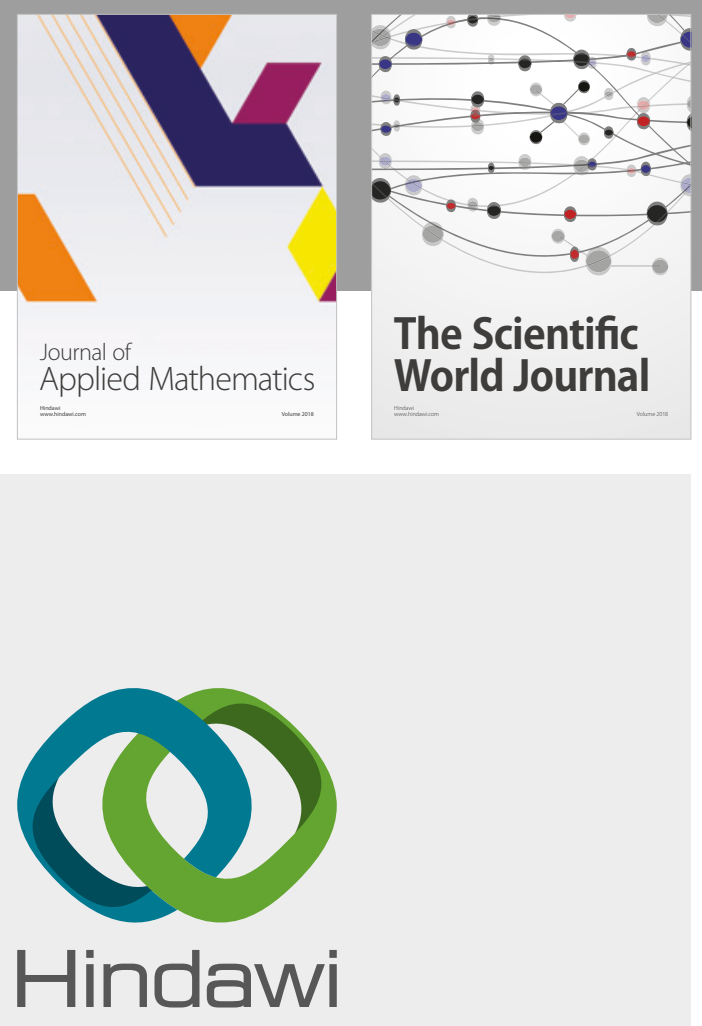

Submit your manuscripts at

www.hindawi.com

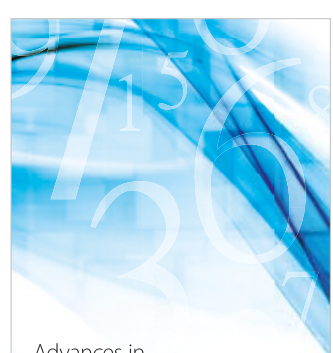

Advances in
Numerical Analysis
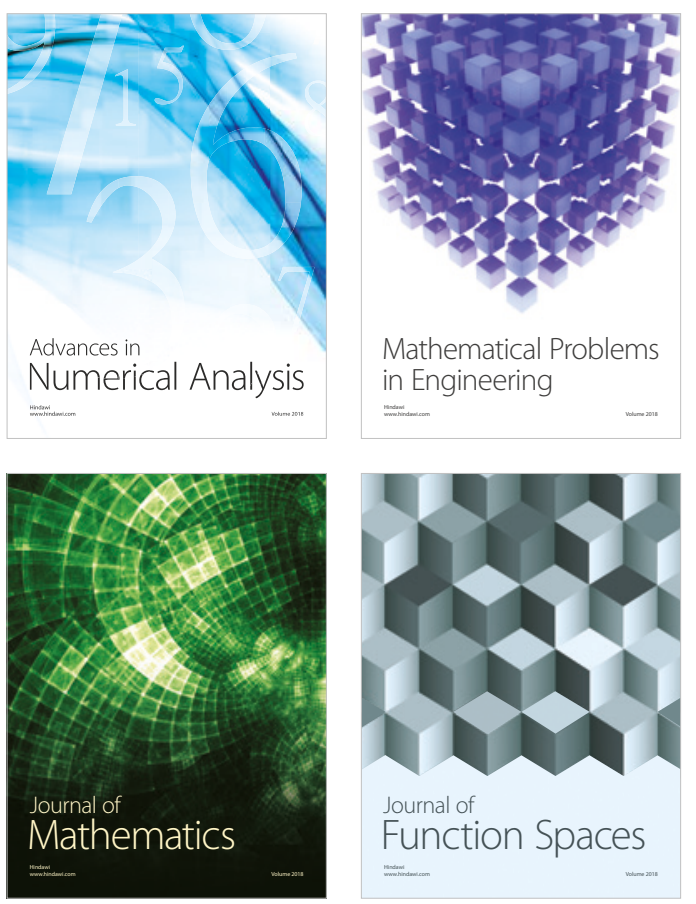

Mathematical Problems in Engineering

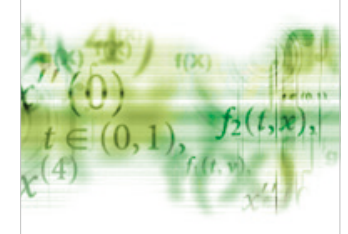

International Journal of

Differential Equations

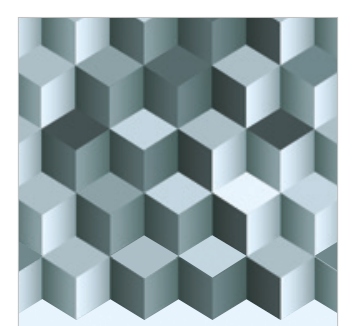

Journal of

Function Spaces
The Scientific

World Journal

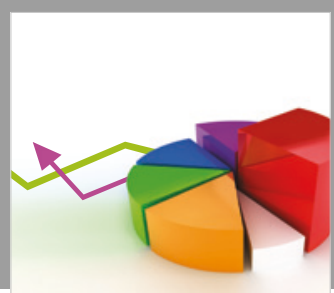

Journal of

Probability and Statistics
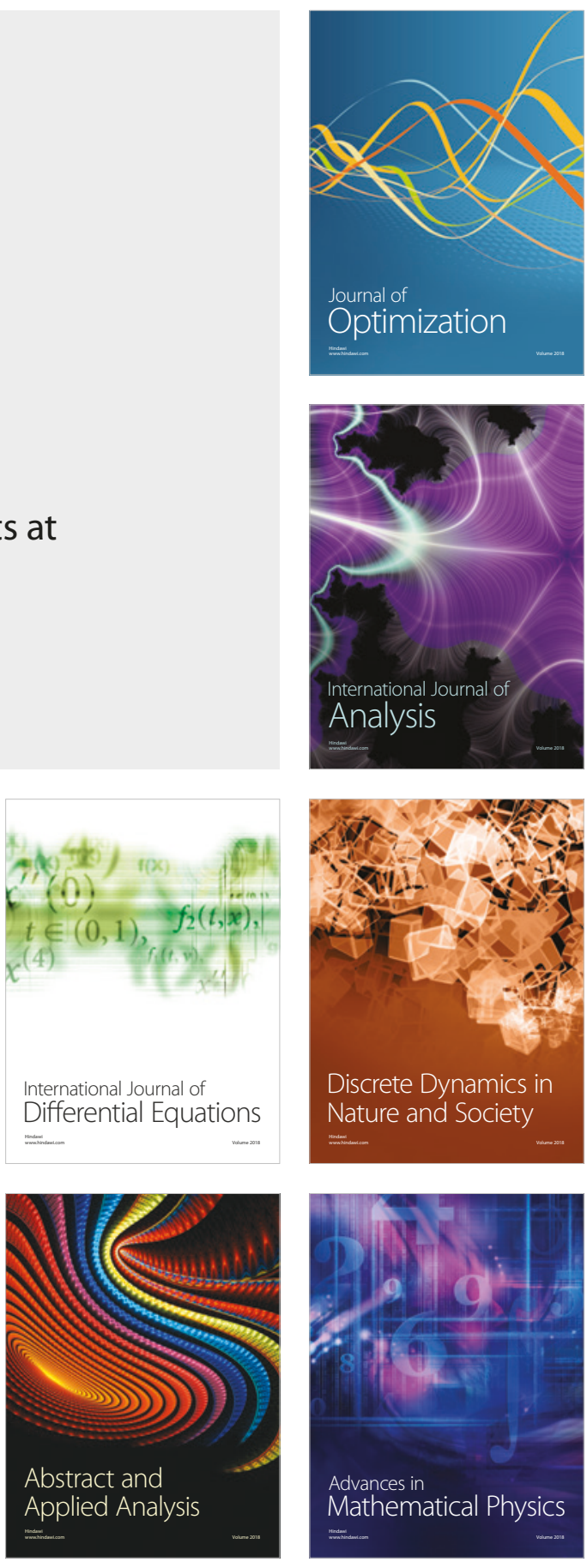